\title{
Deletion of the $\alpha-(1,3)$-Glucan Synthase Genes Induces a Restructuring of the Conidial Cell Wall Responsible for the Avirulence of Aspergillus fumigatus
}

\author{
Anne Beauvais ${ }^{1 *}$, Silvia Bozza ${ }^{2}$, Olaf Kniemeyer ${ }^{3,4}$, Céline Formosa ${ }^{5}$, Viviane Balloy ${ }^{6}$, Christine Henry ${ }^{1}$, \\ Robert W. Roberson7, Etienne Dague ${ }^{5}$, Michel Chignard ${ }^{6}$, Axel A. Brakhage ${ }^{3}$, Luigina Romani ${ }^{2}$, Jean- \\ Paul Latgé ${ }^{1}$
}

1 Unité des Aspergillus, Institut Pasteur, Paris, France, 2 Department of Experimental Medicine and Biochemical Sciences, University of Perugia, Perugia, Italy, 3 Molecular and Applied Microbiology, Leibniz-Institute for Natural Product Research and Infection Biology (HKI), University of Jena, Jena, Germany, 4 Integrated Research and Treatment Center, Center for Sepsis Control and Care Jena, University Hospital (CSCC), Jena, Germany, 5 CNRS, LAAS, Toulouse, France, 6 Unité de Défence Innée et Inflammation, Institut Pasteur, Inserm U874, Paris, France, 7 School of Life Sciences, Arizona State University, Tempe, Arizona, United States of America

\begin{abstract}
$\alpha-(1,3)$-Glucan is a major component of the cell wall of Aspergillus fumigatus, an opportunistic human fungal pathogen There are three genes (AGS1, AGS2 and AGS3) controlling the biosynthesis of $\alpha$ - $(1,3)$-glucan in this fungal species. Deletion of all the three AGS genes resulted in a triple mutant that was devoid of $\alpha-(1,3)$-glucan in its cell wall; however, its growth and germination was identical to that of the parental strain in vitro. In the experimental murine aspergillosis model, this mutant was less pathogenic than the parental strain. The AGS deletion resulted in an extensive structural modification of the conidial cell wall, especially conidial surface where the rodlet layer was covered by an amorphous glycoprotein matrix. This surface modification was responsible for viability reduction of conidia in vivo, which explains decrease in the virulence of triple ags $\Delta$ mutant.
\end{abstract}

Citation: Beauvais A, Bozza S, Kniemeyer O, Formosa C, Balloy V, et al. (2013) Deletion of the $\alpha-(1,3)$-Glucan Synthase Genes Induces a Restructuring of the Conidial Cell Wall Responsible for the Avirulence of Aspergillus fumigatus. PLoS Pathog 9(11): e1003716. doi:10.1371/journal.ppat.1003716

Editor: Chad A. Rappleye, Ohio State University, United States of America

Received March 12, 2013; Accepted August 22, 2013; Published November 14, 2013

Copyright: (c) 2013 Beauvais et al. This is an open-access article distributed under the terms of the Creative Commons Attribution License, which permits unrestricted use, distribution, and reproduction in any medium, provided the original author and source are credited.

Funding: Research in the Aspergillus Unit of JP Latgé was supported by the ESF Fuminomics RNP-06132, ALLFUN FP7- 260338 and ERA-NetPathoGenoMics AntiFun. The funders had no role in study design, data collection and analysis, decision to publish, or preparation of the manuscript.

Competing Interests: The authors have declared that no competing interests exist.

* E-mail: anne.beauvais@pasteur.fr

\section{Introduction}

$\boldsymbol{\alpha}$-(1,3)-Glucan is a major cell wall component of most ascomycetous and basidiomycetous fungi, including the human pathogens that establish their disease upon inhalation of their infective morphotypes (e.g., Paracoccidioides brasilisensis, Histoplasma capsulatum, Blastomyces dermatitidis, Cryptococcus neoformans Aspergillus fumigatus). The role of this polysaccharide during infection has been demonstrated and the mechanisms of its involvement in establishing virulence have been forwarded [1,2]. In C. neoformans, $\alpha-(1,3)-$ glucan anchors the capsule, a well known virulence factor of this fungus, to the yeast cell wall and has been shown to be indirectly associated with virulence since a mutant devoid of $\boldsymbol{\alpha}$ - $(1,3)$-glucan did not have any capsule and, most importantly, was unable to grow at $37^{\circ} \mathrm{C}$ [2]. In the yeast $H$. capsulatum, $\alpha-(1,3)$-glucan was suggested to be essential for virulence because it masked immunogenic molecules: in the $\alpha$-(1,3)-glucan synthase mutant, $\beta$-(1,3)-glucan that is recognized by Dectin- 1 , is exposed at the surface of the cell wall, whereas in the parental strain yeast cells, $\beta$ $(1,3)$-glucan is covered by $\alpha$-(1,3)-glucan, preventing Dectin 1dependent immune response [1].

In A. fumigatus, $\alpha-(1,3)$-glucan accounts for $40 \%$ and $19 \%$ of the mycelial and conidial cell wall polysaccharides, respectively [3]. It is a major adhesive involved in the aggregation of germinating conidia and in biofilm formation [4,5]. Moreover, it has been shown in experimental murine aspergillosis models that $\alpha-(1,3)$ glucan has a prominent immunological function conferring a longterm survival [6]. This immune protection was associated with a reduced neutrophil recruitment in the lungs and reduced inflammatory pathology [6]. $\boldsymbol{\alpha}-(1,3)$-glucan, like conidia, confers a Th1/Treg protection and concomitant Th2 inhibition. These in vivo data were confirmed by in vitro experiments where dendritic cells pulsed with $\alpha$-(1,3)-glucan induced Il12p70 production, a classical Thl promoting cytokine [6]. However, the physiological role of $\alpha$-(1,3)-glucan could not be further investigated in absence of the mutants devoid of $\alpha-(1,3)$-glucan. In A. fumigatus, this polysaccharide is synthesized by three $\alpha$-(1,3)-glucan synthases (Agsp) [3,7]. A triple deletion of the AGS1, AGS2 and AGS3 genes was recently generated in our lab that resulted in an $A$. fumigatus mutant lacking $\alpha$-(1,3)-glucan in the cell wall. In contrast to other fungal pathogens, this triple $A G S A$. fumigatus deletion mutant did not show a distinct growth phenotype in vitro [8].

In the present study, three independently constructed triple ags $1 \Delta$ ags $2 \Delta \operatorname{ags} 3 \Delta(\operatorname{ags} \Delta)$ mutants devoid of $\alpha$-(1,3)-glucan were used to investigate the role of $\alpha-(1,3)$-glucan in A. fumigatus infection. As shown here, the virulence of these $A$. fumigatus triple ags $\Delta$ mutants was extremely attenuated in both immunocompetent and immunocompromised murine models of experimental aspergillosis tested. The defect in virulence correlated with a lack of vegetative fungal dissemination in the lungs, associated with a highly reduced 


\section{Author Summary}

Aspergillus fumigatus is the predominant mold pathogen of humans, responsible for life-threatening systemic infections in patients with depressed immunity. Because of its external localization and specific composition, the fungal cell wall represents a target for recognition by and interaction with the host immune cells. In A. fumigatus, $\alpha$ $(1,3)$-glucan is a key component of the extracellular matrix which encloses the cell wall $\beta-(1,3)$-glucan-chitin fibrillar core. Interestingly, the deletion of the genes responsible for $\alpha$-(1,3)-glucan synthesis resulted in a mutant that exhibited wild type phenotype in vitro; while the altered cell wall organization resulted in this fungus being avirulent in vivo. This study confirms that any modification in the cell wall components is associated with compensatory reactions developed by the fungus to counteract stress on the cell wall that may result in unexpected fungal response when challenged with the host immune system.

inflammation following conidial inoculation. Analysis of the conidia of the triple mutants showed that the lack of virulence of the mutants in vivo was associated to major changes occurring on the cell wall, especially on the surface of the resting and swollen conidia, which resulted in an increased killing by phagocytes.

\section{Results}

\section{The ags $1 \Delta$ ags $2 \Delta \operatorname{ags} 3 \Delta$ (ags $\Delta$ ) mutants are less virulent} than the parental strain in murine model of aspergillosis

In the immunocompetent mice after four days of infection, the number of CFUs of the ags $\Delta$ mutants per lung was much lower than the CFUs per lung of the parental ku80 strain (Fig. 1A; Fig. $\mathrm{S} 1 \mathrm{~A})$. The reduced fungal burden of $\operatorname{ags} \Delta$ was correlated to an absence of inflammation whereas a huge inflammatory response was observed with the parental strain (Fig. 1B, Fig. S1B). This was confirmed by the broncho-alveolar lavage (BAL) analysis, which showed a higher PMN recruitment after infection with kuo0 conidia compared with ags $\Delta$ (Fig. 1C, Fig. S1G). The reduced growth and inflammation in ags $\Delta$ infections was associated with an increase in the expression of the gene coding for the antiinflammatory IL10 and a decreased expression of the gene coding for the pro-inflammatory TNF $\alpha$ in the lungs (Fig. 1D, Fig. S1D). In contrast, ku80 infection was characterized by higher and lower expressions of TNF $\alpha$ and IL10, respectively.

The increased susceptibility of the ags $\Delta$ mutants was confirmed in vitro with murine alveolar macrophages isolated from BAL. After phagocytosis by the isolated macrophages, the killing of the ags $\Delta$ conidia was much higher than the parental strain. The resting conidia of $\operatorname{ags} \Delta$ mutants were killed twice more than the parental strain after $2 \mathrm{~h}$ incubation with the macrophages (Fig. 1E). Further, after $6 \mathrm{~h}$ of incubation, the killing of the mutant reached $60-80 \%$ whereas a maximum of $30 \%$ of the parental strain conidia were killed at this time point (data not shown). Similar difference in the killing ratio between the mutant and parental strains was obtained when the conidia were pre-germinated (swollen conidia; after $6 \frac{1}{2} \mathrm{~h}$ incubation of the conidia in RPMI medium, at $37^{\circ} \mathrm{C}$ ), suggesting that both resting and swollen conidia of the ags $\Delta$ mutants were more susceptible to conidial killing than the parental strain. This twofold increased killing susceptibility of the ags $\Delta$ mutants compared to parental strain did not change in the germinating morphotypes.

In the experimental model of aspergillosis using immunocompromised mice, the virulence of the ags $\Delta$ mutants was also significantly reduced. In a cyclophosphamide model of immunosuppression, infection with the $k u 80$ strain resulted in the mortality of all the mice within 4 days with a high inflammatory response, large foci of pneumonia and exudative bronchiolitis with destruction of bronchi and alveoli, whereas 60 to $80 \%$ mice infected by the ags $\Delta$ mutants survived and did not develop any inflammatory response (Fig. 2A-C, Fig. S2). Similar results were obtained when mice were immunocompromised by the injection of the RB6-8C5 MAb, which depletes circulating PMNs. Inhalation of the $k u 80$ conidia resulted in an extensive pulmonary fungal invasion with high inflammation (Fig. 2D-E). In contrast, in the RB6-8C5 MAb-treated mice lungs, only resting and swollen ags $\Delta$ conidia were observed and their incapability to grow vegetatively culminated in low inflammation (Fig. 2D-E). These results showed that the reduced virulence of the ags $\Delta$ mutant was due to a defect in their conidial survival or vegetative growth in the lung of the infected mice.

\section{Susceptibility of the ags $\Delta$ and parental strain conidia to} antifungal molecules is similar

To investigate the mechanisms responsible for the in vivo growth defect, the germination of $a g s \Delta$ mutant conidia was tested in vitro under stress conditions mimicking the in vivo environment, such as, in the presence of reactive oxidants (ROS), cationic peptides, hypoxia and depletion of iron. The $a g s \Delta$ mutants showed similar growth rates as their parental strain in the presence of Menadione, hydrogen peroxide and Luperox ${ }^{\circledR} 101$ with minimum inhibitory concentrations (MIC) of $30 \mu \mathrm{M}, 10 \mathrm{mM}$ and $2 \mu \mathrm{M}$, respectively (data not shown) irrespective of the $\mathrm{pH}$ of the medium $(\mathrm{pH} 7$ or 4). The killing of resting conidia after $2-6 \mathrm{~h}$ of incubation with macrophages purified from uninfected $\mathrm{p} 47^{\text {phox-1-}}$ mice (depleted in ROS production) were similar to the killing by purified macrophages from uninfected wild type mice (C57BL6 $\mathrm{H}-2^{\mathrm{b}}$ ) (Fig. 2B, data not shown for $6 \mathrm{~h}$ and Fig. 3). These results suggested that the ags $\Delta$ mutant conidia were not more susceptible than the parental strain conidia to reactive oxidants in vitro as well as in vivo. Interestingly, these results also suggested that in our experimental models, conidia from both mutant and parental strains were efficiently killed by ROS-independent mechanisms. Moreover, the absence of iron or the presence of a hypoxic environment did not modify the survival and conidial germination of ags $\Delta$ mutants compared to their parental strain (data not shown). In vitro, the ags $\Delta$ conidia germinated like parental strain conidia in culture medium without supplementation with iron as well as under hypoxic conditions $\left(<1 \%(\mathrm{v} / \mathrm{v}) \mathrm{O}_{2}\right.$ and $9-13 \%(\mathrm{v} / \mathrm{v})$ $\left.\mathrm{CO}_{2}\right)$. The ags $\Delta$ mutants were not more susceptible than the parental strain to cationic peptides. At doses of 230, 100, 40 and $230 \mu \mathrm{g} / \mathrm{ml}$ of Cathelicidin LL-37, $\alpha$ HNP2 and $\beta$ hBD2 defensins and lactoferrin, respectively, no germination differences were seen between parental and mutant strains (data not shown). Similarly, both mutant and parental strain conidial killing was comparable with $0.05 \%$ SDS (data not shown). In addition, no increase in the intracellular labeling of the ags $\Delta$ mutant conidia was seen after incubation with Calcofluor White or FITC (data not shown). These results suggested that the ags $\Delta$ conidia were not more permeable to extracellular toxic molecules than the parental strain. Testing of these different inhibitors in combination (such as $\mathrm{H}_{2} \mathrm{O}_{2}$ or SDS, with Lactoferrin or LL-37) did not result in a differential sensitivity between the parental and mutant strains (data not shown).

These results suggested that, in vitro, the triple ags $\Delta$ mutants were not more susceptible to environmental stresses and antifungal molecules compared to the parental strain. To further investigate the differences in virulence between the mutant and parental 

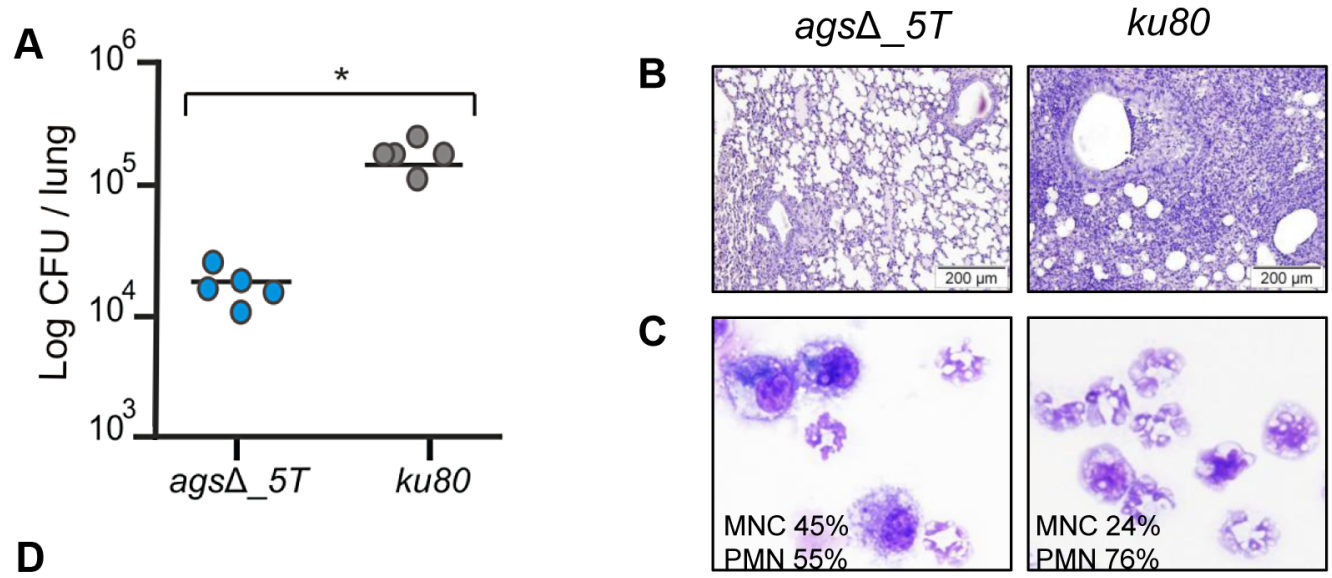

D
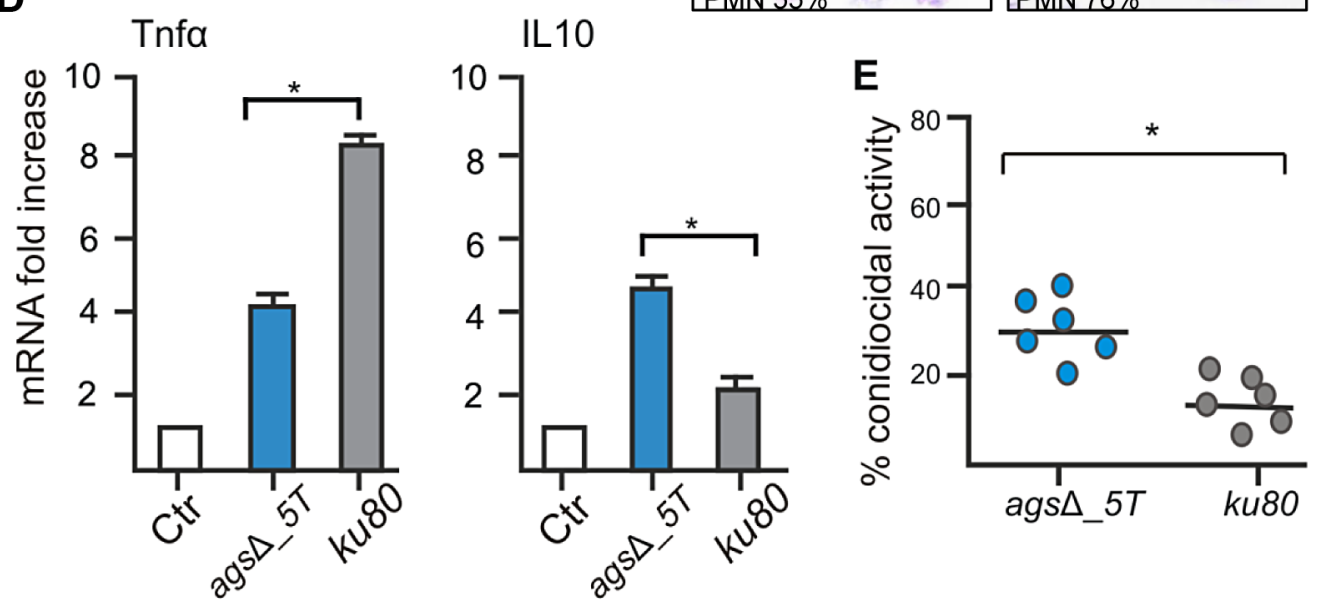

Figure 1. Immunocompetent mice infected with resting conidia of ags $\mathbf{\Delta} \_\mathbf{5 T}$ and parental ( $k u 80$ ) strains. Observations and analysis on mice were done four days post-infection. (A) Fungal load was expressed as log10 CFU/lung. (B) Lung histology (periodic acid-Schiff-staining). Note the polymorphonuclear cells and mononuclear infiltrates surrounding the bronchi in ku80 infected lung. (C) After infection, percentages of monocytes and polymorphonuclear cells found in the lungs alveolar lavage (BAL). (D) Relative expression of TNF $\alpha$ and IL10 assessed by real time RT-PCR on lung total RNA from naïve and infected mice. (E) Conidiocidal activity by purified macrophages from uninfected mice expressed in percentage of CFU inhibition after $2 \mathrm{~h}$ incubation of the conidia with macrophages. Data are representative of at least three independent experiments. Ctl, naïve mice; *, $\mathrm{P}<0.05$.

doi:10.1371/journal.ppat.1003716.g001

strains in vivo, we hypothesize that the killing of the ags $\Delta$ mutant conidia could be due to the induction of an early and strong host immune response towards the mutant conidial morphotypes.

The resting conidia of the $a g s \Delta$ mutants are immediately recognized by the innate immune system because the surface rodlet layer is masked by a layer of glycoproteins

Resting conidia of the ags $\Delta$ mutant were more efficiently phagocytosed by mouse alveolar macrophages than that of the parental ku80 strain. After $1 \mathrm{~h}$ incubation, an average of 3.4 and 1.4 conidia of $a g s \Delta$ mutants and ku80 were engulfed per macrophage, respectively (Fig. 4, Fig. S3). This result suggested that the ags $\Delta$ mutant and parental strain conidial surfaces are different. To investigate such structural modifications, conidial surfaces were imaged by atomic force microscopy (AFM). In contrast to the $k u 80$ conidia that are covered with a crystalline-like array of rodlets [9], the ags $\Delta$ mutant conidial surface was amorphous without any organized structure (Fig. 5A). The presence of an amorphous material covering the surface of the ags $\Delta$ conidia was further confirmed by TEM (Fig. 5B).

To investigate if the rodlet layer is still present on the ags $\Delta$ mutant conidial surface but masked by this amorphous material, $k u 80$ and ags $\Delta$ resting conidia were treated with hydrofluoric acid $(\mathrm{HF})$ to extract the rodlet protein. Similar amount of the hydrophobic RodA protein, which constitutes the rodlet layer, could be extracted from the ags $\Delta$ and parental strain conidia $\left(26.7 \pm 4.9 \mu \mathrm{g}\right.$ and $26.5 \pm 3.0 \mu \mathrm{g}$ per $10^{9}$ conidia, respectively). Figure $5 \mathrm{C}$ shows that the two bands, $16 \mathrm{kDa}$ and $14.5 \mathrm{kDa}$ of RodAp classically seen from HF treatment of the conidia [10] were present in the SDS-PAGE profiles of ags $\Delta$ and $k u 80$ resting conidial HF-extracts. These data confirmed AFM and TEM observations that on the ags $\Delta$ mutant conidial surface the rodlets were present but hidden by an amorphous material.

Because of the presence of this amorphous material covering the hydrophobic rodlets, we asked whether the observed surface changes correlated with differences in conidial adhesive properties. To understand this, we mapped and quantified the nanoscale adhesion properties of $k u 80$ and ags $\Delta$ mutant conidia by AFM using bare Si3N4 tip. Figure 6 (and Fig. S4) showed that the presence of this unorganized material on the ags $\Delta$ mutant conidial surface was associated with a dramatic reduction in their conidial surface adhesive properties. For the parental strain, force-distance curves recorded across the cell surface revealed large adhesion forces, with a magnitude of $0.6 \pm 0.039 \mathrm{nN}$ as shown by the 

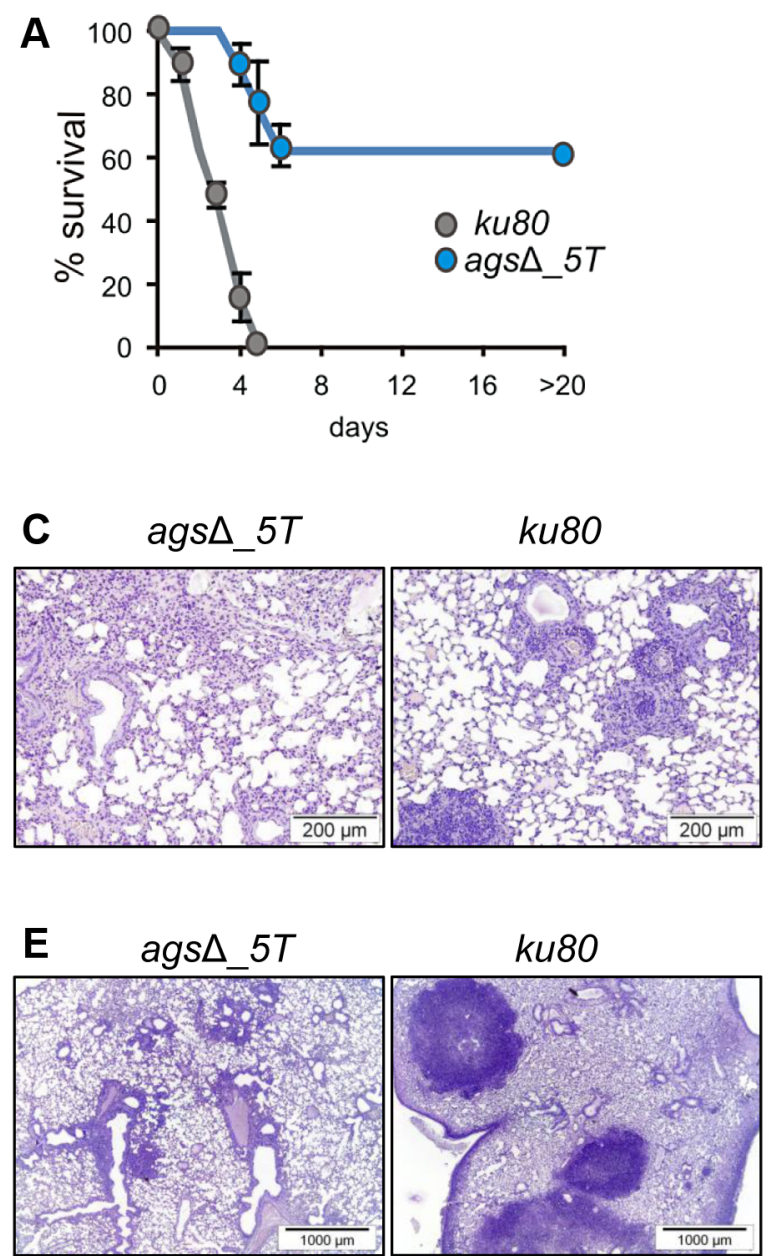
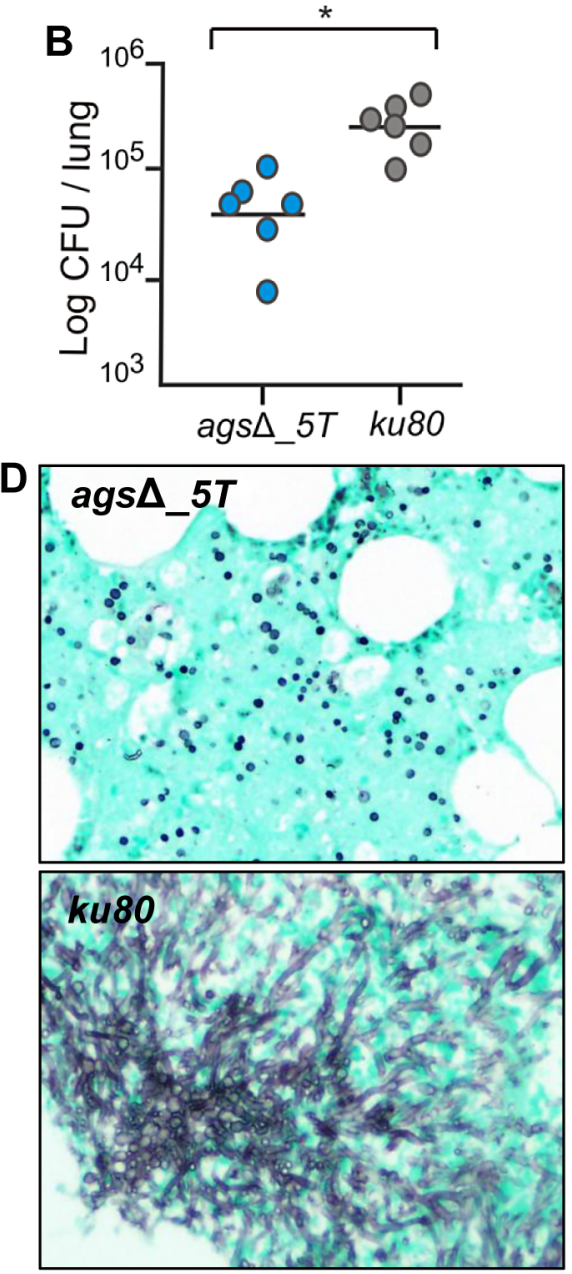

Figure 2. Cyclophosphamide immunosuppressed mice and anti-Ly6G treated neutropenic mice infected with resting conidia of ags $\Delta$ 5T and parental (ku80) strains. (A-C) Cyclophosphamide immunosuppressed mice; (D-E) anti-Ly6G treated neutropenic mice; (A) Survival (\%) and (B) fungal growth estimated as CFUs in lung. (C and E) lung histology (periodic acid-Schiff-staining). Note the polymorphonuclear cells and mononuclear infiltrates surrounding the bronchi in ku80 infected lung. (D) Histological appearance of lungs of anti-Ly6G neutropenic mice infected with conidia of $a g s \Delta \_5 T$ and ku80 (Gomori's methanamine silver-staining). Note the absence of mycelial development of ags $\Delta \_5 T$ conidia in neutropenic mice. Data are representative of at least three independent experiments. ${ }^{*}: p<0.05$.

doi:10.1371/journal.ppat.1003716.g002

adhesion force histogram (Fig. 6A-G). In contrast, structural changes in ags $\Delta$ conidia caused profound modifications of the cell surface physico-chemical properties (Fig. 6D-F, Fig. S4). Forcedistance curves showed the absence of adhesion forces over the entire surface of the mutant conidia. This decrease in the ags $\Delta$ conidial adhesion capacities indicated a modification of the cell surface hydrophobicity that could have influenced conidial phagocytosis.

Further, chemical nature of the amorphous layer present on the ags $\Delta$ mutant conidial surface was investigated. It was not composed of polysaccharides since the labeling of $\beta$-(1,3)-glucan with the $\beta$-(1,3)-glucan receptor GNBP3, chitin with WGA, galactomannan (GM) with an anti-GM monoclonal antibody and galactosaminogalactan (GAG) with an anti-GAG monoclonal antibody were negative (data not shown). In contrast, a strong labeling of the resting ags $\Delta$ conidium with ConA was observed suggesting that the surface layer was rich in glyco-conjugates (Fig. 7).

To extract these amorphous surface materials, ags $\Delta$ resting conidia were incubated in $0.5 \mathrm{M} \mathrm{NaCl}$ for $2 \mathrm{~h}$ and the extracted materials were positive for protein assay. As shown in the Figure 8 (and Fig. S5), incubation with $\mathrm{NaCl}$ did not release any proteins from the parental ku80 strain whereas the extracts from ags $\Delta$ mutant conidia contained $160 \mu \mathrm{g}$ proteins per $10^{10}$ conidia. It was verified that the amorphous glycoprotein layer was removed after $\mathrm{NaCl}$ treatment because ConA labeling on the conidia after $\mathrm{NaCl}$ treatment was negative (data not shown). Further, extracted protein mixture was subjected to proteomic analysis. Thirty-four proteins were identified and in-silico analysis of these proteins by SigPred (http://www.cbs.dtu.dk/services/SignalP/) and CADRE (http://www.cadre-genomes.org.uk/Aspergillus_fumigatus/) revealed that all of them had a signal peptide except Sodl (AFUA_5G09240, [11]) (Table 1, Table S1). Most of these proteins were hydrolases and the most abundant protein was a putative $\beta$ - $(1,4)$-glucan hydrolase (AFUA_7G06140). Other glycosylhydrolases were hexosidases or $\mathrm{N}$-acetylhexosaminidases (AFUA_1G05770; AFUA_1G14560, AFUA_1G10790, AFUA_8G05020, AFUA_6G10730). A unique aspartic phosphatase was identified that was different from the one previously identified as a major mycelial cell wall protein [12]. Three 


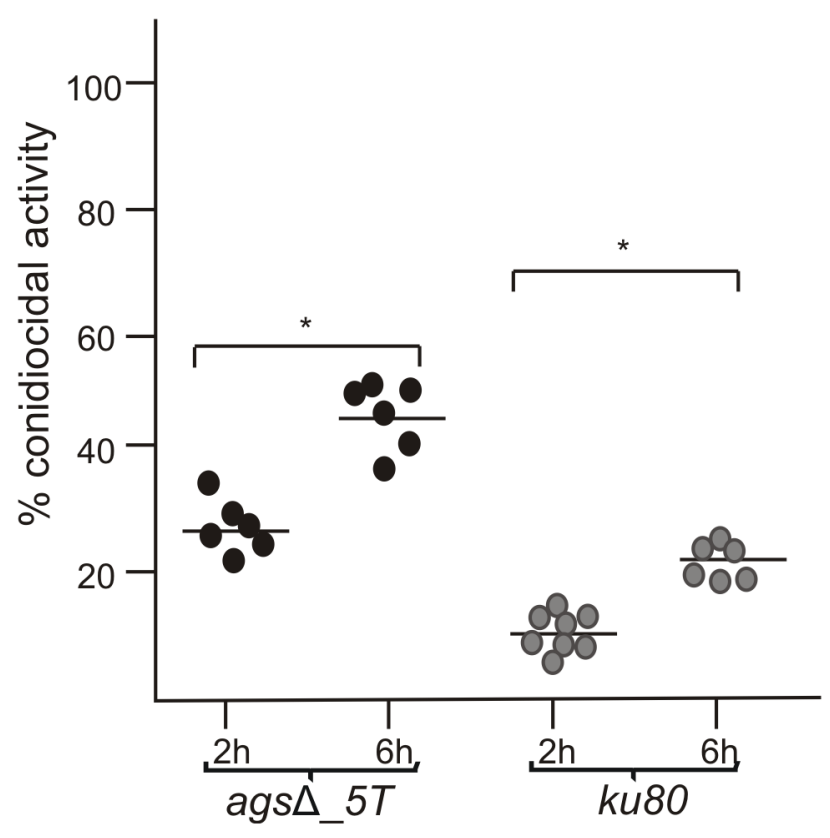

Figure 3. Conidiocidal activity of macrophages isolated from uninfected $\mathrm{p} 47^{\text {phox-1- }}$ mice against resting conidia of ags $\Delta \_5 T$ and parental (ku80) strains. Conidiocidal activity is expressed in percentage of CFU inhibition after 2 and $6 \mathrm{~h}$ incubation of the conidia with macrophages. Data are representative from at least three independent experiments. ${ }^{*}, \mathrm{P}<0.05$.

doi:10.1371/journal.ppat.1003716.g003

peptidases (AFUA_2G03510,AFUA_4G03490,AFUA_8G04120) and the two aspartic proteases, Peplp and Pep2p (AFUA_5G13300, AFUA_3G11400), known to be associated with the conidial cell wall were found [13]. Two well known allergens of $A$. fumigatus were also detected (Aspf1 (AFUA_5G02330) and Aspf13 (AFUA_2G12630) [14]). Other protein such as oxidoreductases and enzymes of sugar metabolism (pyruvate dehydrogenase kinase AFUA_2G11900 and isopropylmalate dehydrogenase AFUA_1G15780) were present in lower amount as they were identified only once or twice in the proteomic survey. Interestingly, Sodlp and RodAp (AFUA_5G09580), known to be highly expressed in resting conidia [11], were also found in this $\mathrm{NaCl}$ extract. A similar SDS-PAGE profile was obtained when urea/thiourea buffer was used to extract ags $\Delta$ conidial surface material, indicating that the proteins recovered were not depending on the extraction buffer (data not shown). The fact that many proteins were present above the surface rodlet layer suggested that in contrast to the parental strain, the lack of $\alpha 1,3$ glucan has led to a different cell wall retainment of these glycoproteins in the ags $\Delta$ mutant conidia.

In vitro analysis of the cytokines produced during the first $5 \mathrm{~h}$ of incubation with alveolar macrophages showed that high amounts of pro-inflammatory $\mathrm{TNF} \alpha$ cytokine were produced upon interaction with ags $\Delta$ mutant conidia whereas no $\mathrm{TNF} \alpha$ was produced when the parental strain was incubated with macrophages under the same incubation conditions (Fig. 9A, Fig. S6A). Stimulation of the macrophages with the ags $\Delta$ conidial $\mathrm{NaCl}$ extract also induced TNF $\alpha$ expression (Fig. 9B; Fig. S6B). These results suggested that the surface glycoprotein layer on the resting ags $\Delta$ conidia was responsible for the induction of pro-inflammatory cytokine production immediately after conidial phagocytosis.

Thus, the deletion of the $A G S$ genes resulted in an unexpected modification of the mutant conidial surface with the emergence of

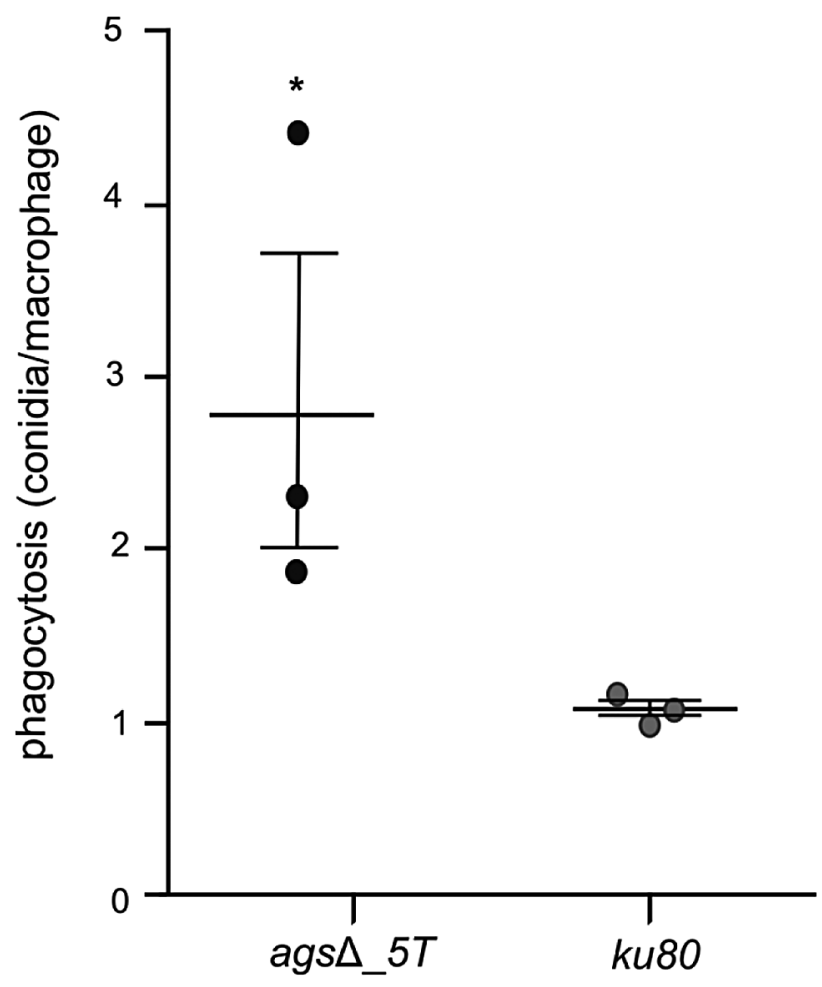

Figure 4. Phagocytosis activity by isolated macrophages from uninfected mice against resting conidia of ags $\Delta \_5 T$ and parental (ku80) strains. Index of phagocytosis is expressed in number of conidia per alveolar macrophage after $1 \mathrm{~h}$ incubation of conidia with macrophages. Data are representative of at least 3 independent experiments. ${ }^{*}, \mathrm{P}<0.05$.

doi:10.1371/journal.ppat.1003716.g004

an amorphous layer on the resting conidial surface over the rodlet layer, which altered biophysical properties, consequently affecting conidial interaction with the host immune system.

Polysaccharide PAMPS are exposed on the surface of the swollen conidia of the triple ags $\Delta$ mutants

Increased cytokine production seen in the macrophages over a 5 h-time period could also come from changes occurring at the surface of germinating conidia since it has been shown previously that conidia starts germinating intracellularly in the macrophage lysosome after the first $2 \mathrm{~h}$ of phagocytosis [15]. In addition, Figure 2 shows that $a g s \Delta$ conidia undergo swelling in the infected lungs before being killed. The structural changes of the early germ tubes resulting from the $A G S$ deletion were investigated by cytochemistry. The swollen conidia of the triple ags $\Delta$ mutants presented an increased labeling by WGA compared to the parental strain (Fig. 10A and data not shown). In addition, swollen ags $\Delta$ conidia were positive with the $\beta$ - $(1,3)$-glucan receptor GNBP3, whereas both resting and swollen conidia of the parent strain were negative (Fig. 10B and data not shown). In contrast, there were no differences in the immunolabeling of the swollen conidia of parental and $a g s \Delta$ mutants with anti-GAG and anti-GM monoclonal antibodies (Fig. S7). These results suggest that the absence of $\alpha$-(1,3)-glucan that normally hides $\beta$-(1,3)-glucan and chitin, exposes these PAMPs at the surface of the swollen ags $\Delta$ conidia. These results were also in agreement with the chemical analysis of the cell wall: the mycelium cell wall of the ags $\Delta$ contained 1.7 and 2 times more chitin and $\beta$-(1,3)-glucan, respectively, than the cell wall of the parental strain [8]. 

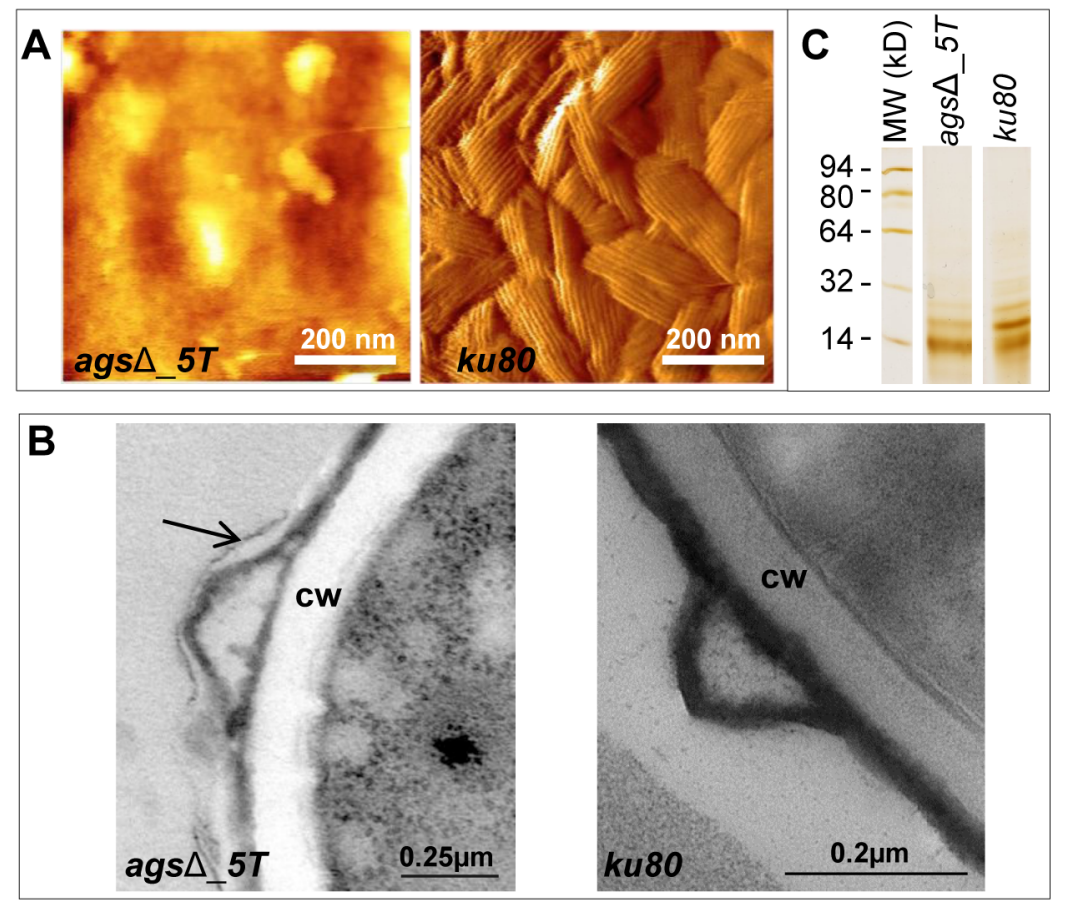

Figure 5. Surface analysis of resting conidia of ags $\Delta \_5 T$ mutant and parental $(k u 80)$ strains. (A): height images $(z$-range $=1 \mu \mathrm{m}$; recorded in water with silicon nitride tips). Atomic Force Microscopy (AFM) images showing the amorphous surface without the rodlet layer on the triple ags $\Delta \_5 T$ mutant conidia whereas the rodlet are observed on the parental strain conidial surface. (B): TEM observations. Note the presence of an extracellular material on the surface of the $a g s \Delta \_5 T$ conidia (arrow); CW: cell wall. (C): SDS-PAGE (15\% gel) of Hydrofluoric acid (HF) extracts of rodlets from resting conidia showing the two bands, $16 \mathrm{kDa}$ and $14.5 \mathrm{kDa}$ of RodAp classically seen from HF treatment of the conidia [10]. Data are representative of at least three independent experiments.

doi:10.1371/journal.ppat.1003716.g005

Figure 11 represents a model to explain the sequential immune events upon inhalation of the ags $\Delta$ mutant and parental strain conidia and their differential impact/in vivo fate based on our in vitro assays as well as in vivo experiments using murine aspergillosis models. The presence of glycoproteins hiding the rodlet layer increases the phagocytic rate and promotes an immediate host immunological response towards the triple ags $\Delta$ mutants during phagocytosis. Once the mutant conidium is internalized, the conidial swelling results in an increased exposure of PAMPs on the swollen $a g s \Delta$ conidial surface. Such surface modifications further boosts pre-existing host defense induced by the resting ags $\Delta$ conidia. In contrast, the resting conidium of the parental strain are not recognized by the phagocytes and do not display major PAMPs on the surface of the conidium during the intracellular swelling. Since ags $\Delta$ conidia did not seem more sensitive to host antifungal molecules compared to the parental strain, we hypothesize that differences in the killing in the later growth stages resulted from an early and enhanced host response induced by the modified surface of the resting ags $\Delta$ conidia. This early stimulation will be responsible for the killing of the germinating ags $\Delta$ conidia. On the contrary, in the partially immunosuppressed experimental murine models, limited and delayed killing of the parental strain conidia enables their further vegetative growth.

\section{Discussion}

In this study we showed that the $\operatorname{ags} \Delta$ mutants displayed a reduced virulence associated with an inhibition of germination in vivo and a reduction of the inflammatory response after $24 \mathrm{~h}$ infection (decreased TNF $\alpha$ and increased IL10 expressions and reduced recruitment of PMNs). The low level of $\mathrm{TNF} \alpha$ seen with the triple ags $\Delta$ mutants fits with the lack of recruitment of neutrophils seen with this mutant after $24 \mathrm{~h}$ infection. However, during our in vitro experiments with macrophages incubated during $5 \mathrm{~h}$ with $\operatorname{ags} \Delta$ or ku80 conidia, we observed the induction of proinflammatory cytokines. This indicated that the lack of inflammation seen at later stages of infection in mice was due to the inhibition of vegetative growth of the ags $\Delta$ mutants rather than a failure to stimulate inflammation. This was in agreement with the fact that $a g s \Delta$ conidia were killed before their hyphal development.

The primary phenotype of the resting conidia of the ags $\Delta$ mutants was the absence of visible rodlet layer on the conidial surface. Even though the rodlets were present in the mutant conidia, their masking by a (glyco-)protein layer restored the immune sensing that is usually silenced when the rodlets are present on the surface of the wild type conidia $[10,16]$. The ags $\Delta$ conidia were covered by proteins, which are usually secreted during vegetative growth. Most hydrolases found in the additional amorphous surface layer of the resting $\operatorname{ags} \Delta$ conidia were usually identified during mycelial growth in a protein-based medium $[14,17]$. How these proteins are able to cross the conidial cell wall remains an open question. Their presence on the surface is certainly due to the modifications of the cell wall integrity resulting from the three $A G S$ deletions. Interestingly, in three independent $\mathrm{HF}$ extractions, the amount of $14.5 \mathrm{kDa}$ RodAp was slightly higher than the $16 \mathrm{kDa} \operatorname{RodAp}(20-23 \% 16 \mathrm{kDa} \operatorname{RodA}$ in ags $\Delta$ mutants compared to $40-50 \%$ in the parental strain; Fig. $5 \mathrm{C}$ ) suggesting that the rodlet structure of the mutant was less organized than the rodlet of the parental strain, which putatively modified the ionic strength of the hydrophobin layer in the ags $\Delta$ mutants [18]. Such structural modifications may affect the adherence of the hydrophilic glycoproteins to rodlets through 

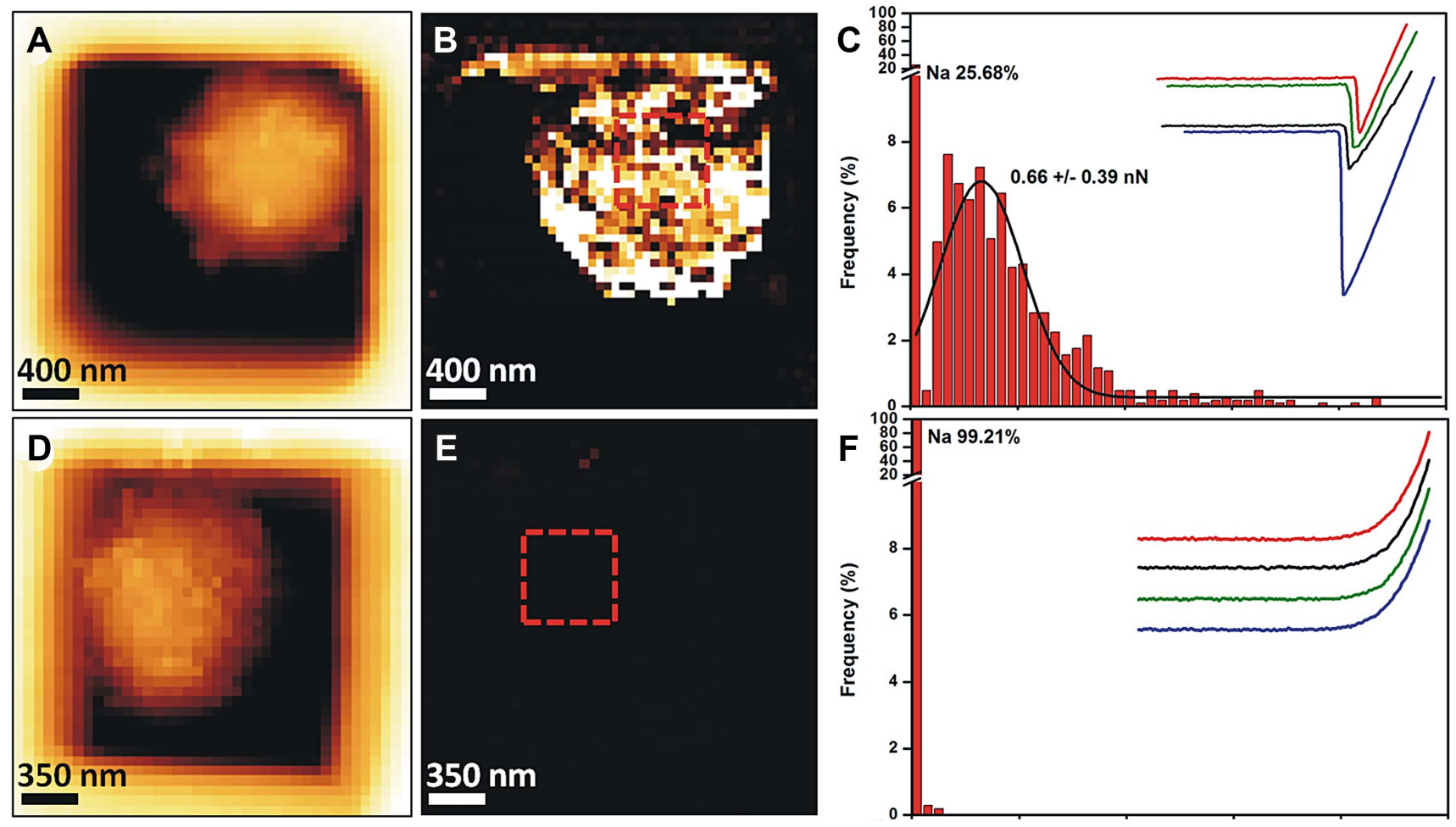

Adhesion Force $(\mathrm{nN})$

Figure 6. Imaging and adhesive properties of $A$. fumigatus resting conidia of the parental strain and ags $\Delta 5 T$ mutant. Structural changes of ags $\Delta \_5 T$ correlate with a loss of cell surface adhesive properties. (A-C) parental strain; (D-F) ags $\Delta \_5 T$ mutant; (A, D) height images (zrange $=1 \mu \mathrm{m}$; recorded in water with silicon nitride tips); $(B, E)$ adhesion force maps (z-range: $5 \mathrm{nN})$ corresponding to the height image; $(C, F)$ Representative force-distance curves and adhesion force histograms $(n=1024)$ recorded on the surface of parental strain $(C)$ and ags $\Delta \_5 T(F)$. doi:10.1371/journal.ppat.1003716.g006

electrostatic binding, since these proteins were easily extracted by salt. How these glycoproteins reached the surface of the cell wall is still not understood. This should not be related to changes in cell wall permeability since the ags $\Delta$ mutants were not more permeable to FITC or drugs that affect viability such as ROS, cationic peptides or Calcofluor White than the parental strain (data not shown). Alternatively, the hydrolases, because of their enzymatic activity, may harm the cell wall structure itself and this would help the proteins to cross the cell wall barrier. The stimulation of the expression of $\mathrm{TNF} \alpha$ after incubation with macrophages (isolated from naive mice $\mathrm{BAL}$ ) with ags $\Delta$ mutant conidial $\mathrm{NaCl}$ extract showed that these proteins located on the conidial surface were sensed first by the immune system and were able to induce an immediate immune response towards ags $\Delta$ conidia. It was previously shown that some of these surface proteins are recognized by $\mathrm{T}$ cells and can induce a Thl protective response [6]. In particular, the secreted aspartic protease Pepl that has been found in $\mathrm{NaCl}$ extract from ags $\Delta$ conidia conferred protection against infection, associated with a reduced neutrophil recruitment in BAL and a reduced inflammatory pathology in the lung. Hiding of the rodlet layer by an amorphous glycoprotein layer that stimulates the host response is not exclusively specific to
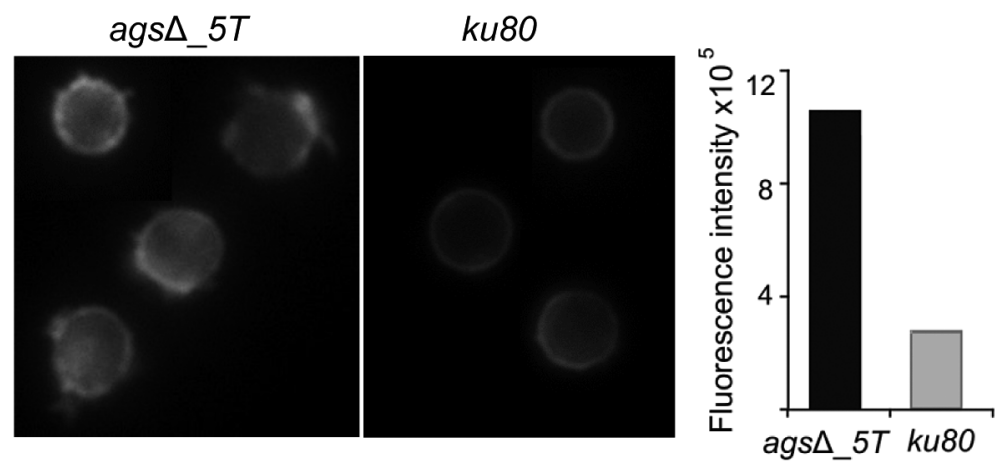

Figure 7. ConA-FITC labeling of ags $\Delta \_5 T$ mutant and parental strain ( $\left.k u 80\right)$ resting conidia. Note the increase in the ConA labeling on the $a g s \Delta \_5 T$ mutant conidial surface. Histograms represent the calculated fluorescence intensity of the corresponding images, expressed in Einstein per seconds.

doi:10.1371/journal.ppat.1003716.g007 


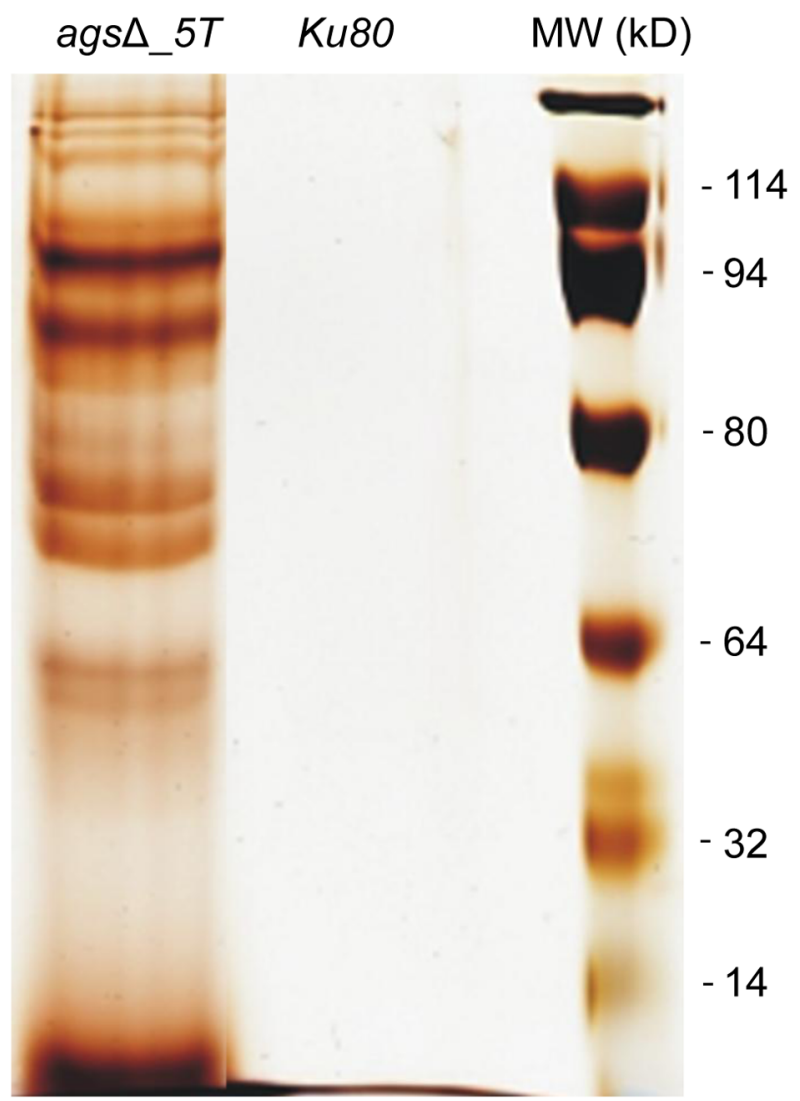

Figure 8. $\mathrm{NaCl}$ extracted proteins from the surface of ags $\Delta$ _5T resting conidia. SDS-PAGE (10\% gel) of proteins extracted after $2 \mathrm{~h}$ incubation of $a g s \Delta \_5 T$ and ku80 resting conidia in $0.5 \mathrm{M} \mathrm{NaCl}$. doi:10.1371/journal.ppat.1003716.g008

the ags $\Delta$ deletion, since a similar conidial phenotype was observed on chitin synthase mutants $[19,20]$. Similarly, in $B$. dermatitidis, the absence of $\alpha$-(1,3)-glucan at the surface of the yeast increased the expression of W1-1 adhesin/antigen that were bound to phagocytic cells and suppressed the generation of the pro-inflammatory cytokine TNF $\alpha[21,22]$.

The exposure of polysaccharide PAMPs on the surface of germinating conidia consecutively to triple $A G S$ deletions also plays a role in stimulating the host innate immune response and inducing the production of antifungal molecules by the innate immune cells. The exposure of $\beta$ - $(1,3)$-glucan at the surface of germinating ags $\Delta$ conidia will favor a Dectin-1-mediated host response [23]. Similarly, increased $\beta$-(1,3)-glucan exposure due to caspofungin treatment stimulated the host defense reaction against A. fumigatus $[24,25]$. In addition, the positive binding of WGA and ConA also suggested that other receptors such as the mannose or/ and chitin/N-acetylglucosamine, which are known to stimulate an antifungal response, can also be involved in this modified immune response [26]. Similar to the situation with the $\operatorname{ags}_{s} \Delta$ mutants, it was shown that the lack of $\alpha-(1,3)$-glucan in $H$. capsulatum also led to the unmasking of PAMPs [1]. The protective role of $\alpha$-(1,3)-glucan has been also shown in $B$. dermatitidis and $P$. brasiliensis where the absence of $\alpha$-(1,3)-glucan at the surface of the yeast and/or its replacement by $\beta$-(1,3)-glucan stimulated the host defense reaction $[21,27]$. Recently, the masking of chitin by $\alpha$ - $(1,3)$-glucan has been shown to be essential for the virulence of the plant pathogen Magnaporthe grisea [28].
The molecules responsible for the killing of the ags $\Delta$ conidia remain unknown. However, it is clear that ROS were not responsible for the differences in killing between the ags $\Delta$ mutants and the parental strain conidia since the ags $\Delta$ mutants did not display a higher sensitivity to ROS in vitro and the killing of ags $\Delta$ conidia was similar in $\mathrm{p} 47^{\mathrm{phox}-/-}$ mice compared to C57BL/6 (Fig. 3). Although a link between increased oxidative response and enhanced damage to A. fumigatus has been repeatedly demonstrated in the past [29,30], recent studies, especially with chronic granulomatous disease (CGD) patients, have shown that NADPHindependent mechanisms can contribute to Aspergillus killing as much as ROS [31,32]. Among possible mechanisms of NADPHindependent activity, D'Angelo et al. [33] have suggested that defensins and cathelicidins, known for their role in host defense, could be responsible for $A$. fumigatus killing in CGD mice. This seems however not the case for the ags $\Delta$ mutants as our in vitro studies indicated that the ags $\Delta$ mutants did not show a higher susceptibility to cathelicidin LL-37 or HNP2 and hBD2 defensins. Modification of the conidial surface may also lead to an increased binding of Surfactant Proteins A and D, Mannose Binding Lectin $\mathrm{C}$ or Penthraxin 3 that are known to be associated to an increased phagocytosis and an activation of the complement pathway known to play a major role in the killing of A. fumigatus [21,34,35,36,37]. Based on our data, it remains impossible to infer the killing of the ags $\Delta$ mutant conidia to currently known antifungal immune defense mechanisms. It can also be postulated that the killing may be due to an early burst of unknown toxic molecules or that the killing is the result of several antifungal molecules acting synergistically [38]. Our cell wall analysis suggested also that the cell wall architecture is perturbed in the inner as well as in the outer layer and that this perturbation may result in modifications of the cell wall permeability to specific antifungal molecules [8]. These could be responsible for an increased susceptibility of the $\operatorname{ags} \Delta$ mutant to the host defense molecules.

The story of $A$. fumigatus $\alpha$-(1,3)-glucan remains a two-sided coin. In the wild type strain, $\alpha-(1,3)$-glucan induces an anti-A. fumigatus response as the injection of this polysaccharide into mice was immunoprotective and obviously responsible for the production of a Thl response that is directed against $A$. fumigatus [6]. It could be expected that their removal favors the virulence of the mutant. In reality, the opposite happens due to the reorganization of the cell wall of the resting and germinating conidia upon triple $A G S$ deletions. The presence of glycoproteins hiding the rodlet layer and the exposure of PAMPs in the germinating conidia modified the immunological response of the host, which increased phagocytosis and killing of the ags $\Delta$ mutants, and induced proinflammatory cytokine production. It is the structural modification of the entire cell wall consecutive to the $A G S$ deletions that is responsible for an early stimulation of the host defense reactions. Interestingly, these structural modifications did not modify the survival of the fungus in vitro but are essential for the in vivo survival. The difference in the surface composition of the resting and swollen conidia of the ags $\Delta$ mutants led to an immediate sensing of the immunogenic molecules resulting in an early response of the phagocyte towards the ags $\Delta$ conidia. The deleterious effect of a delayed immune response on the microbial virulence is well known.

The $\alpha$-(1,3)-glucan study tells us that the deletion of one cell wall gene does not lead only to the disappearance of the product of the encoded gene but results in a complete restructuration of the fungal cell wall. This has been shown with the deletion of the $A G S$ genes in this study but also with other cell wall genes or consecutively to the use of antifungals acting on the cell wall in several fungal species [39]. Such structural and chemical 
Table 1. Proteins identified in the $\mathrm{NaCl}$ extract of $a g s \Delta \_5 T$ and $a g s \Delta \_n 8$ conidia.

\begin{tabular}{|c|c|c|c|}
\hline AFUA number & Common Name of Target & Known Gene & MW (Kd) \\
\hline AFUA_7G06140 & Putative secreted 1,4- $\beta$-D-glucan glucanhydrolase & & 78.38 \\
\hline AFUA_6G10130 & Putative N,O-diacetyl muramidase & & 24.64 \\
\hline AFUA_1G05770 & $\beta$-glucosidase ExoG2 & EXOG2 & 94.75 \\
\hline AFUA_3G07520 & Exo $\beta$-1,3-glucanase & & 86.72 \\
\hline AFUA_2G01240 & Putative $\beta$-fructofuranosidase & & 57.26 \\
\hline AFUA_1G14560 & Putative $\alpha$-1,2-mannosidase, MsdS & & 53.84 \\
\hline AFUA_1G10790 & Putative $\alpha$-1,2-mannosidase & & 92.7 \\
\hline AFUA_8G05020 & Putative secreted $\alpha$-N-acetylhexosaminidase NagA & & 57.4 \\
\hline AFUA_4G01290 & Glycosyl hydrolase family 75 chitosanase & & 25.1 \\
\hline AFUA_5G13300 & Secreted aspartic endopeptidase Pep1 & PEP1 & 41.6 \\
\hline AFUA_3G11400 & Secreted aspartic endopeptidase Pep2 & PEP2 & 43.3 \\
\hline AFUA_4G03490 & Putative secreted tripeptidyl-peptidase TppA, SedB & $S E D B$ & 65.83 \\
\hline AFUA_2G03380 & Putative alkaline serine protease & & 13.4 \\
\hline AFUA_2G03510 & Putative pheromone processing carboxypeptidase Sxa2 & & 49.75 \\
\hline AFUA_8G04120 & Secreted serine carboxypeptidase S1 & SCP1 & 61.28 \\
\hline AFUA_3G07030 & Putative secreted glutaminase GtaA & & 76.15 \\
\hline AFUA_2G12630 & allergenic cerato-platanin Aspf13, serine alkaline protease & ASPF13 & 15.94 \\
\hline AFUA_5G02330 & allergenic restrictocin, mitogilin Aspf1 & ASPF1 & 19.59 \\
\hline AFUA_4G03660 & Putative acid phosphatase, $\mathrm{PhoB}$ regulated & & 46.1 \\
\hline AFUA_5G09240 & $\mathrm{Cu}, \mathrm{Zn}$ superoxide dismutase Sod 1 & SOD1 & 16.36 \\
\hline AFUA_3G03450 & Putative oxidoreductase & & 58.58 \\
\hline AFUA_3G08070 & GMC oxidoreductase & & 67.61 \\
\hline AFUA_2G04200 & 4-hydroxyphenylpyruvate dioxygenase, HppD & $H P P D$ & 45.53 \\
\hline AFUA_4G13000 & Putative amine oxidase & & 119 \\
\hline AFUA_4G07690 & Putative phosphoribosylaminoimidazolecarboxamide formyltransferase & & 65 \\
\hline AFUA_1G16420 & Uncharacterized protein & & 58.55 \\
\hline AFUA_5G09580 & hydrophobin RodA & RODA & 16.17 \\
\hline AFUA_1G15780 & Putative 3-isopropylmalate dehydrogenase Leu2A & & 39 \\
\hline AFUA_2G11900 & Putative pyruvate dehydrogenase kinase & & 49.43 \\
\hline AFUA_6G07980 & Putative cyclin-dependent protein kinase & & 36.65 \\
\hline AFUA_4G03630 & Putative sterol 24-c-methyltransferase & & 42.57 \\
\hline AFUA_1G11000 & Putative $\mathrm{C} 6$ transcription factor & & 82.26 \\
\hline AFUA_1G00700 & hypothetical protein & & 150.55 \\
\hline AFUA_3G06520 & conserved hypothetical protein & & 65.71 \\
\hline
\end{tabular}

Identification was done by MS/MS and MS with a mascot score above a threshold of 54. Details are showed in Table S1.

doi:10.1371/journal.ppat.1003716.t001

modifications in the cell wall will have an obvious impact on the immune response of the host towards the corresponding mutant. Our study also suggests, any interpretation stating that the immune response towards a cell wall mutant is only due to the lack of the product of the deleted gene should be considered with care $[40,41]$.

\section{Materials and Methods}

\section{Strains and culture conditions}

All strains were grown in $2 \%(\mathrm{w} / \mathrm{v})$ malt agar slants and 1 weekold conidia were recovered from the slants by vortexing with $0.05 \%$ (v/v) Tween 20 aqueous solution. Swollen conidia and germ tubes were produced after $5 \mathrm{~h}$ and $10 \mathrm{~h}$, respectively, after incubation at $37^{\circ} \mathrm{C}$ in Brian's medium (Brian) [42]
The A. fumigatus parental strain $\mathrm{AkuB}^{\mathrm{ku} 80} \Delta \mathrm{pyrG}$ (ku80, [43]) and three ags $\Delta$ mutant strains independently obtained: ags $1 \Delta$ ags 2 $\Delta$ ags3 $\Delta \_5 T\left(a g s \Delta \_5 T\right)$ obtained previously [8] and two new ones, ags $1 \Delta$ ags $2 \Delta a g s 3 \Delta n 8$ and $a g s 1 \Delta a g s 2 \Delta a g s 3 \Delta \_n 6.2 \quad$ (ags $\Delta \_n 8$ and ags $\left.\Delta \_n 6.2\right)$, were used in this study. Since it had been impossible to complement ags $\Delta$ mutant for reasons explained previously [8], two new triple ags $\Delta$ mutants were constructed independently using the strategy described previously to exclude the possibility that undesired mutations had occurred during the deletion process. The lack of $\alpha$-(1,3)-glucan in the cell wall of mutant strains was confirmed by both chemical and immunolabeling assays (Fig. S8). Chemical analysis of the cell wall was performed as previously described [44]. For immunolabeling assays, 5-10 h germinated conidia were labeled using the MOPC 104E monoclonal antibody, which binds specifically to $\alpha$-(1,3)-glucan [45] (Beauvais A. Institut 

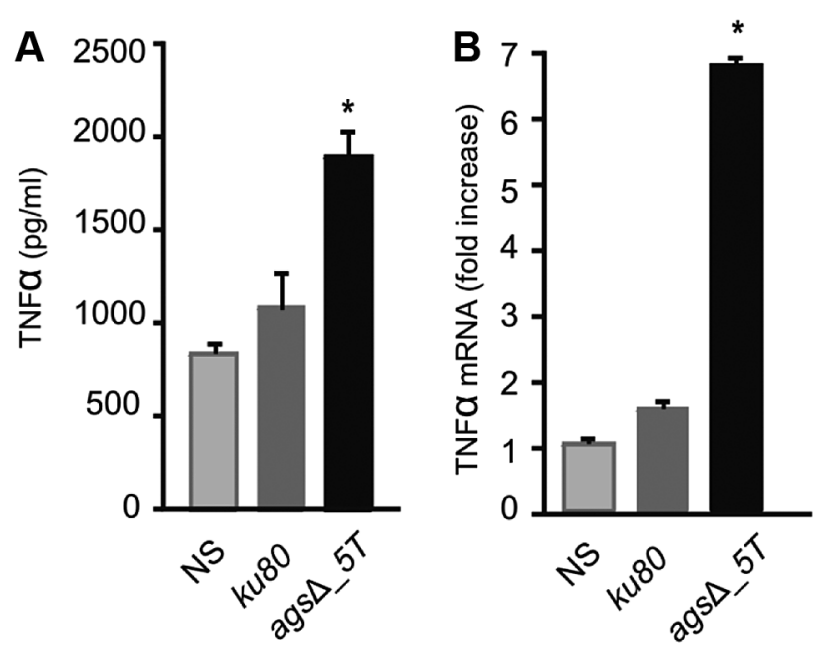

Figure 9. TNFa production or expression by macrophages (isolated from uninfected immunocompetent mice) upon interaction with resting conidia of parental ( $k u 80)$ and ags $\Delta 5 T$ strains or ags $\Delta \_5 T$ conidial $\mathrm{NaCl}$ extract $(3.2 \mu \mathrm{g}$ proteins) respectively. (A) TNF $\alpha$ was quantified after $5 \mathrm{~h}$ incubation of the conidia with macrophages; (B) Relative expression of TNF $\alpha$ assessed by real time RT-PCR in total RNA from macrophages after $5 \mathrm{~h}$ incubation of the ags $\Delta \_5 T$ conidial $\mathrm{NaCl}$ extract with macrophages. $\mathrm{NaCl}$ supernatant from $k u 80$ resting conidia incubated for $2 \mathrm{~h}$ in $0.5 \mathrm{M} \mathrm{NaCl}$ was used as a control. NS: Non-stimulated. ${ }^{*}, \mathrm{P}<0.05$.

doi:10.1371/journal.ppat.1003716.g009

Pasteur, Paris, France, unpublished results). Paraformaldehyde (PFA) fixed swollen and germinating conidia were permeabilized prior to immunolabeling as previously described [46]. MOPC 104E (Sigma) and control mouse IgM (Sigma) were used at a dilution of 1:25 and the goat antimouse IgG-TRITC $(\mathrm{H}+\mathrm{L}$, Sigma) was used as the secondary antibody at a dilution of 1:50.
The three triple mutants used in this study germinated, sporulated and conidiated like the parental strain in vitro (data not shown, [8]).

Analysis of the conidial surface

Conidial surface was analyzed by Atomic Force Microscopy (AFM). The sample immobilization is achieved by assembling the living conidia within the patterns of microstructured, functionalized poly-dimethylsiloxane (PDMS, Sylgard 184) stamps using convective/capillary deposition [47]. Images and force measurements were performed in deionised water, respectively in contact mode and in Quantitative Imaging (QI) mode and Force Volume (FV) mode. For both experiments we used bare MLCT AUWH cantilever (nominal spring constant $0.01 \mathrm{~N} / \mathrm{m}$ ) (Bruker). Single cells were first localized and imaged and then switched over to QI and FV modes to record adhesion force maps. AFM Nanowizard II and III (JPK Instruments, Berlin, Germany) were used to capture the images. The cantilevers spring constants were measured by the thermal noise method [48] ranging from 0.0160 to $0.0190 \mathrm{~N} / \mathrm{m}$. Force curves were analyzed in order to determine the adhesion force between the conidia and the AFM tip. These adhesions were plotted as bright pixels, brighter colors indicating larger adhesion values. For each strain, images that were obtained for at least three conidia from independent cultures and analyzed with different tips, were representative of the entire conidial population inside each mutant and parental strain. The results acquired on the spores were analyzed on JPK Data Processing software.

The rodlet layer was extracted from the spore surface by incubating $10^{9}$ dry conidia with $48 \%(\mathrm{v} / \mathrm{v})$ hydrofluoric acid $(\mathrm{HF})$ for $72 \mathrm{~h}$ at $4^{\circ} \mathrm{C}$. The contents were centrifuged $(10,000 \mathrm{rpm}$, $10 \mathrm{~min}$ ) and the supernatant obtained was dried under $\mathrm{N}_{2}$. The dried material was reconstituted in $\mathrm{H}_{2} \mathrm{O}$ and an aliquot was subjected to $15 \%(\mathrm{w} / \mathrm{v})$ SDS-PAGE analysis and visualized by silver nitrate staining. Bands were quantified using Image lab software (BioRad).
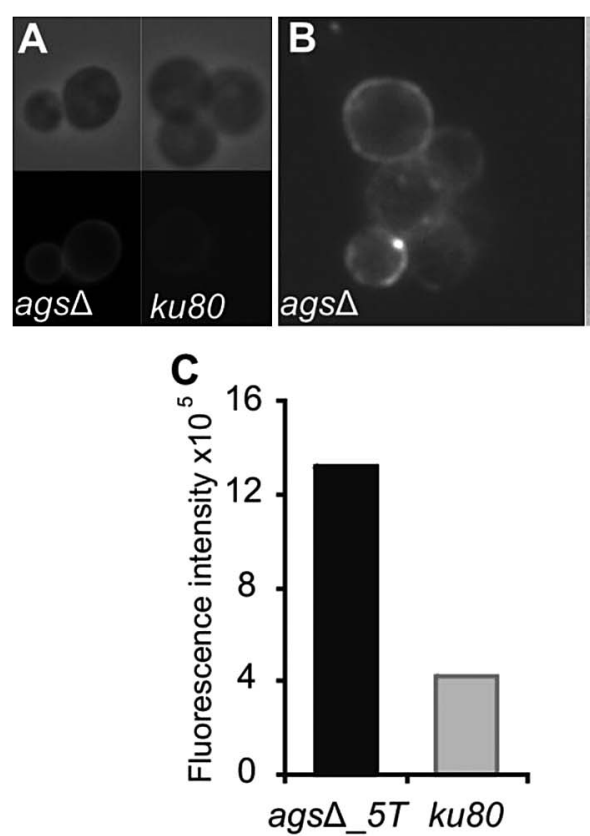

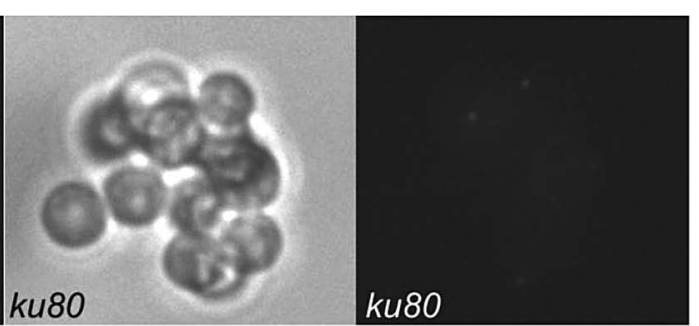

D

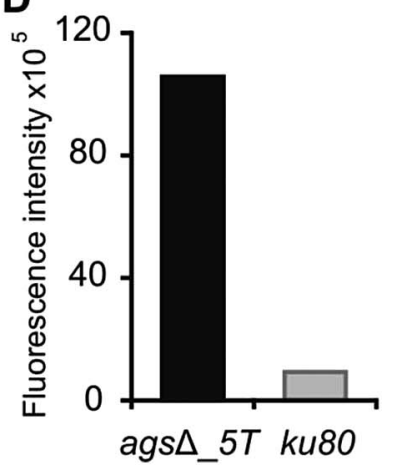

Figure 10. Labeling of the surfaces of ags $\Delta$ _5T and parental strain swollen conidia by WGA and the $\beta(1,3)-g l u c a n$ receptor GNBP3. The surfaces of the swollen conidia were labeled by WGA-FITC (A) and GNBP3 (B) as described in material and methods. (C, D) Histograms represented the calculated fluorescence intensity of the corresponding images ( $A$, $B$ respectively), expressed in Einstein per seconds. doi:10.1371/journal.ppat.1003716.g010 
Immediate phagocytosis and activation of the immune response induced by the glycoprotein $\downarrow$ layer covering the rodlets
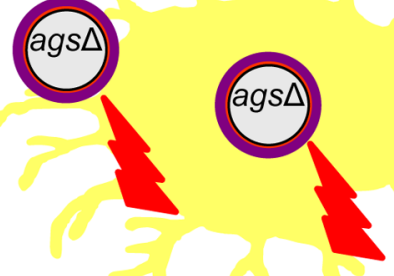

Increased PAMPs $(\beta-(1,3)$-glucan? and chitin (N) exposure during conidial swelling reinforcing the $\downarrow$ activation of the immune response

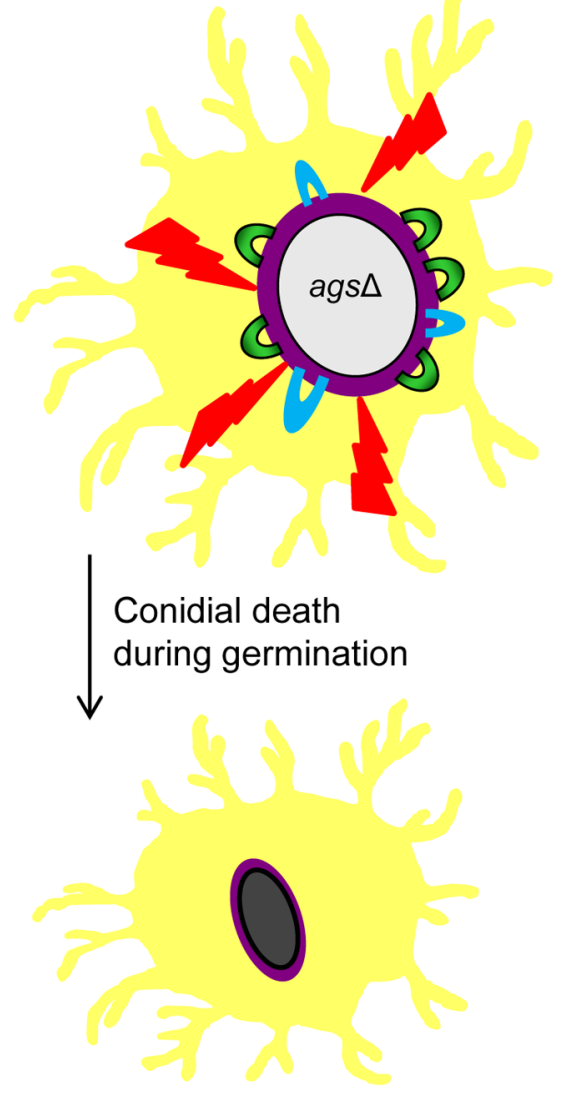

Time

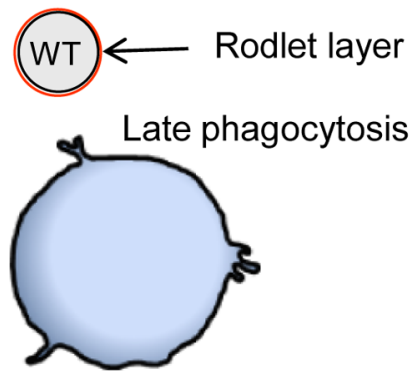

Lack of recognition as a result of the rodlets

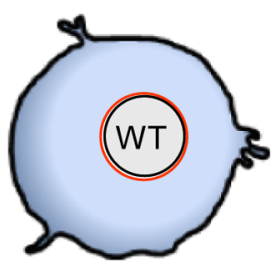

Swelling of the conidia, exposure of the $\alpha-(1,3)$ glucan, but delayed immune response too $\downarrow$ late to kill all conidia

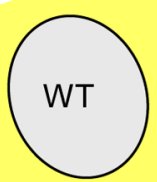

Moderate exposure of the PAMPs during conidial germination, but too late $\downarrow$ to kill the fungus.

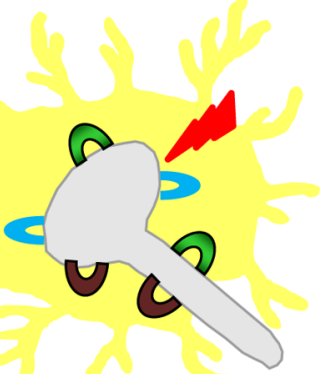

Figure 11. Working model explaining sequential and differential immune events upon inhalation of the ags $\Delta$ mutant and the parental ( $k$ u80) strain conidia. The presence of the glycoprotein layer on the triple ags $\Delta$ mutant conidial surface hides the rodlet layer. Increased exposure of PAMPs (WGA and ConA positive molecules and $\beta-(1,3)$-glucans) during vegetative growth in the triple ags $\Delta$ mutant modifies the host immunological response. This facilitates phagocytosis and killing of the triple ags $\Delta$ mutant and stimulates pro-inflammatory immune responses. doi:10.1371/journal.ppat.1003716.g011 
To analyze the components present on the surface, conidia were incubated in $0.5 \mathrm{M} \mathrm{NaCl}$ solution for $2 \mathrm{~h}$ at room temperature at a ratio of $10^{10}$ conidia per ml. The $\mathrm{NaCl}$ supernatant was recovered after centrifugation and directly subjected to $10 \%$ SDS-PAGE (w/ v). The protein concentrations in the extracts were determined by the Coomassie brilliant blue method [49], using BioRad kit and BSA as the standard. Proteomic analysis of the $\mathrm{NaCl}$ extract was carried out as described previously with slight modifications [50]. A total amount of 50-100 $\mu \mathrm{g}$ protein was loaded onto IPG strips (11 cm, pH 3-7; GE Healthcare Life Sciences) by in-gel rehydration. After equilibration of the IPG strips, SDS-gel electrophoresis was carried out using Criterion AnykD TGX gels (Bio-RAD). Proteins were visualised by colloidal Coomassie staining [51]. After scanning, gel images were analysed with the software Delta 2D 4.3. (Decodon). Protein spots were excised and analysed by mass spectrometry using an ultrafleXtreme MALDI-TOF/TOF device (Bruker Daltonics).

\section{Fluorescence microscopy}

Resting and swollen conidia were PFA-fixed $(2.5 \%(\mathrm{v} / \mathrm{v})$ PFA in PBS) for one night at $4^{\circ} \mathrm{C}$, washed three times with $0.1 \mathrm{M} \mathrm{NH}_{4} \mathrm{Cl}$ in PBS, once with PBS and then incubated with different antibodies or lectins as described previously [52].

Galactosaminogalactan (GAG) was labeled with a monoclonal mouse antibody as described previously [53] $(20 \mu \mathrm{g} / \mathrm{ml})$ and a mock monoclonal antibody was used as a control. The secondary goat anti-mouse IgG-TRITG (Sigma) antibody was used at a dilution of 1:200.

Galactomannan was labeled with a rat anti-Galactofuranose (Galf) monoclonal antibody (EBA2, diluted 1:1000, a kind gift of M. Tabouret from BioRad, Steenvorde [54]). Control Rat monoclonal antibody of the same isotype and the secondary goat anti-rat FITC (Sigma-Aldrich) antibody were used at a dilution of $1: 1000$ and 1:500, respectively.

$\beta$-(1,3)-glucan was labeled with the $\mathrm{N}$-terminal $\beta$-(1,3)-glucan binding domain of Drosophila pattern recognition receptor, GNBP3 (homologous to Mammalian Dectin 1) at a concentration of $3 \mu \mathrm{g} /$ $\mathrm{ml}$ and a polyclonal mouse antiserum against GNBP3 at 1:200 dilution (kind gifts from A. Roussel, CNRS, Orleans and D. Ferrandon, CNRS, Strasbourg, France [55]). Goat anti-mouse IgG FITC 1:200 diluted (Sigma) was used as secondary antibodies.

The glucosamine moiety of chitin/chitosan and mannose/ glucose moieties of glycoproteins and glucans were labeled respectively with WGA-FITC and ConA-FITC (Sigma) at $0.1 \mathrm{mg} / \mathrm{ml}$ concentrations upon incubating the conidia for $15 \mathrm{~min}$ at lab temperature.

\section{Susceptibility to oxidative stress conditions, Lactoferrin, Cathelicidin LL-37, HNP2 and hBD2 defensins, absence of iron and hypoxia}

Stress conditions induced by Menadione $(0$ to $30 \mu \mathrm{M})$ and 2,5$\operatorname{Bis}\left(\right.$ tert-butylperoxy)-2,5-dimethylhexane $\quad$ (Luperox $\left.{ }^{\circledR} 101\right) \quad(0$ to $2 \mathrm{mM}$ ) were tested on both parental and mutant $A$. fumigatus strains grown on agar-RPMI (RPMI 1640, Sigma without glutamine) supplemented with $1 \%$ agar (Difco), $0.3 \mathrm{~g} / 1 \mathrm{~L}-$ glutamine and $0.1 \mathrm{M}$ MOPS or MES (to obtain a $\mathrm{pH}$ of 7 or 4, respectively) at $37^{\circ} \mathrm{C}$ for $24-48 \mathrm{~h}$.

Stress conditions induced by Lactoferrin $0.45-231 \mu \mathrm{g} / \mathrm{ml}$ (Sigma) or Cathelicidin LL-37 $0.45-231 \mu \mathrm{g} / \mathrm{ml}$ (Sigma), SDS $\left(0.006-0.2 \%\right.$; Merck) and $\mathrm{H}_{2} \mathrm{O}_{2}(0.003-0.1 \%$; Fluka) were tested on $A$. fumigatus strains grown on Brian medium without supplementation with iron or RPMI-glutamine-MOPS medium (described above) [38]. Combinations of $0.05 \%$ SDS or $0.012 \%$
$\mathrm{H}_{2} \mathrm{O}_{2}$ and Lactoferrin or Cathelicidin LL37 at concentrations of $231 \mu \mathrm{g} / \mathrm{ml}$ were tested in the same media, as described in Clavaud et al [38].

Stress condition induced by HNP2 $(100 \mu \mathrm{g} / \mathrm{ml}$; Sigma $)$ and hBD2 (25 $\mu \mathrm{g} / \mathrm{ml}$; Sigma) defensins were also tested by incubating $10^{6}$ conidia/ml with the defensins for $10-16 \mathrm{~h}$ at $37^{\circ} \mathrm{C}$ in RPMIglutamine-MOPS medium.

The growth of $A$. fumigatus strains was tested in Brian medium without supplementation with iron at $37^{\circ} \mathrm{C}$ and under hypoxia conditions using AnaeroGen sachet (Oxoid), which reduces the oxygen level in a jar to below $1 \%$ that results to a $\mathrm{CO}_{2}$ level between $9-13 \%$.

\section{Transmission electron microscopy (TEM)}

Aliquots $(20 \mu \mathrm{l})$ of concentrated conidia were placed onto a Formvar-coated nickel or gold mesh grids, which were then placed between the flat sides of two B-type brass planchets (Ted Pella Inc., Redding, CA). The grids were used as spacer creating a thin layer of cells that allows higher yields of well-frozen cells. The samples were immediately frozen with liquid nitrogen under high pressure (2,100 bar) using a Bal-Tec HPM 010 high pressure freezing machine (Bal-Tec Products, Middlebury, CT, USA). Following cryofixation, the samples were freeze-substituted at $-85^{\circ} \mathrm{C}$ in $1 \%$ glutaraldehyde (Electron Microscopy Sciences, Washington, PA, USA) and 1\% tannic acid in acetone for $72 \mathrm{~h}$. After, the samples were rinsed thoroughly with three changes of fresh acetone at $-85^{\circ} \mathrm{C}$ for a total of $45 \mathrm{~min}$. Cells were infiltrated with $1 \% \mathrm{OsO}_{4}$ in acetone for $1 \mathrm{~h}$ at $-85^{\circ} \mathrm{C}$ before being slowly warmed to room temperature over $5 \mathrm{~h}$. The cells were then rinsed in acetone and slowly infiltrated with and polymerized in Spurr's resin. Embedded cells were cut into serial $70 \mathrm{~nm}$ thick sections with an Ultracut R Microtome (Leica, Vienna, Austria) and collected on Formvarcoated copper slot grids. Sections were post-stained with $2 \%$ uranyl acetate in $50 \%$ ethanol for $5 \mathrm{~min}$ followed by $5 \mathrm{~min}$ with Sato's lead citrate [56]. The grids were carbon-coated and viewed at $80 \mathrm{kV}$ using a JEOL 1200EX transmission electron microscope (JEOL USA, Inc., Pleasanton, CA, USA).

\section{Analysis of ags $\Delta$ mutant virulence}

Female 8- to 10-week-old inbred C57BL6 (H-2 ${ }^{\mathrm{b})}$ mice were obtained from Charles River Breeding Laboratories (Calco, Italy). Experiments were performed according to the Italian Approved Animal Welfare Assurance A-3143-01. Breeding pairs of homozygous $\mathrm{p} 47^{\text {phox- } /-}$ mice, raised on C57BL6 background, were purchased from Harlan Laboratories and bred under specificpathogen free conditions at the breeding facilities of the University of Perugia, Perugia, Italy [33]. Infections were performed on one model of immunocompetent mice and in two different models of invasive pulmonary aspergillosis as previously described [6]. In the first immunosuppressed model, mice were subjected to intraperitoneal administration of cyclophosphamide $(150 \mathrm{mg} / \mathrm{kg}$ body weight) one day before infection as described previously [6]. In the second immunosuppressed model, mice were treated with antiLy6G monoclonal antibody (clone RB6-8C5 MAb; eBienscience; $100 \mu \mathrm{g} /$ mouse) administered intra-peritoneally one day before infection. Rat anti-E. coli $\beta$-galactosidase (clone GLL 113) was used as a control IgG. Treatment with the anti-Ly6G MAb is known to selectively deplete mature neutrophils, eosinophils and dendritic cells [57] and at $24 \mathrm{~h}$ after administration, the number of circulating neutrophils dropped to $20 \pm 12 / \mathrm{mm}^{3}$ compared to $1120 \pm 227 / \mathrm{mm}^{3}$ in controls, and the treated mice continued to be low for circulating neutrophils counts up to 5-days. Mice were monitored for survival and fungal growth (determined as colony forming unit (CFU) per organ) four days post-infection as 
described previously [58]. All mice underwent necropsy for histopathological observation of fungal burden in the lungs four days post-infection. For histology, sections $(3-4 \mu \mathrm{m})$ of paraffinembedded lungs were stained following periodic acid-Schiff (PAS) protocol. Collection of the bronchoalveolar lavage (BAL) fluid and the morphometry [\% monocytes (MNC) or polymorphonuclear (PMN) cells] was performed after four days infection as previously described [6]. Total and differential cell counts were performed after staining BAL smears with May-Grünwald Giemsa reagents (Sigma) before analysis. At least 200 cells per cytospin preparation were counted and the absolute number of each cell type was calculated. Cytospin preparations were observed using a BX51 microscope (Olympus, Milan, Italy). Histology images were captured using a high-resolution DP7 1 camera (Olympus).

For phagocytosis and conidiocidal activity, alveolar macrophages from uninfected mice were isolated from BAL as described [15]. For phagocytosis, macrophages were incubated at $37^{\circ} \mathrm{C}$ with unopsonized FITC (Sigma) labeled conidia [59] at an effector to conidial ratio of 5:1, for $1 \mathrm{~h}$ in RPMI medium in micro-chambers (Ibitreat). Unbound conidia were removed by washing with RPMI and cells were fixed with $3 \%(\mathrm{v} / \mathrm{v})$ PFA for $1 \mathrm{~h}$ in PBS. After fixation, the cells were incubated with a rabbit polyclonal antiFITC antibody (Invitrogen) diluted 1:2000 and a secondary rabbit antibody conjugated to Alexafluor 568 (dilution, 1:2000) (Invitrogen). This last procedure labels only cell surface-associated conidia and the ingested conidia remained unlabeled. The number of ingested conidia per macrophage was determined on 200 macrophages. For conidiocidal activity, macrophages isolated from uninfected C57BL6 $\left(\mathrm{H}-2^{\mathrm{b}}\right)$ and $\mathrm{p} 47^{\text {phox- } /-}$ mice were incubated at $37^{\circ} \mathrm{C}$ with unopsonized resting or swollen conidia $\left(6 \frac{1}{2} \mathrm{~h}\right.$ in $\mathrm{RPMI}$ at $\left.37^{\circ} \mathrm{C}\right)$, at an effector to fungal cell ratio of $1: 10$, for 2-6 hours in an ELISA plate wells. After removing the supernatant, Triton X100 (1\%) was added to the wells and incubated at $37^{\circ} \mathrm{C}$ for $10 \mathrm{~min}$ to lyse the macrophages and to collect phagocytized conidia. The percentage of phagocytized conidia capable of further germination was determined by spotting phagocytized conidia (at suitable dilution) on a nutritive agar medium and counting those conidia capable of forming germ tube among spotted conidial population. We verified that the use of Triton X100 to lyse macrophage did not affect conidial germination as the percentage of germinations were similar $(97 \pm 1 \%)$ for the ags $\Delta \_5 T$, ags $\Delta \_n 6.2$, ags $\Delta \_n 8$ mutants and the parental strain with or without Triton-treatment. The differences in the germination of the conidia from the stock solution used for macrophage conidicidal activity study permitted the calculation of conidiocidal activity.

For cytokine quantification, total RNA was extracted from lungs of immunocompetent mice four days post-infection, or from macrophages isolated from BAL fluid of uninfected mice and incubating with ags $\Delta \mathrm{NaCl}$ extracts containing $3.2 \mu \mathrm{g}$ proteins, for $5 \mathrm{~h}$. The cytokines expressed and productions were quantified by Real-time PCR and ELISA, respectively as described previously [6].

Statistical significance was analyzed by one- or two-way ANOVA or paired t-test with Prism software (GraphPad software, San Diego, CA) and $p$-values $\leq 0.05$ were considered to be significant. Data were representative of at least two independent experiments or pooled from three to five experiments. The in vivo groups consisted of six mice/group and experiments were repeated at least three times. Macrophage experiments were done three times with three different batches of macrophages and conidia.

All experiments were performed using the ags $\Delta \_5 T$ (Figs. 1-10, Table 1, Table S1). Virulence and proteomic analyses were performed also using ags $\Delta \_n 8$ (Figs. S1, S2, S3, S4, S5, S6, S7, S8,
Table 1 and Table S1). Major phenotypes and virulence data were verified with ags $\Delta \_n 6.2$ (Figs. S1, S5, S6, S7, S8).

\section{Ethics statement}

Mouse experiments were performed according to the Italian Approved Animal Welfare Assurance 245/2011-B. Legislative decree $157 / 2008-B$ regarding the animal license was obtained by the Italian Ministry of Health lasting for three years (2008-2011). Infections were performed under avertin anesthesia and all efforts were made to minimize suffering.

\section{Supporting Information}

Figure S1 Immunocompetent mice infected with resting conidia of ags $\Delta$ triple mutants and parental kuso strain. Observations and analysis on mice were done four days post-infection. (A) Fungal CFUs in lungs infected with conidia of ags $\Delta \_5 T$, ags $\Delta \_n 6.2$, ags $\Delta \_n 8$ and ku80. (B) lung histology (periodic acid-Schiff-staining) and $(\mathrm{C})$ Percentages of monocytes and polymorphonuclear cells found in the lung alveolar lavage (BAL) of mice infected with conidia of ags $\Delta \_n 6.2$ and ags $\Delta \_n 8$ mutants (periodic acid-Schiff-staining and Gomori's methanamine silver-staining) (D) Relative expression of TNF $\alpha$ and IL10 assessed by real time RT-PCR of the total RNA extracted from the lungs of naive and mice infected with conidia of ags $\Delta \_n 6.2$ and ags $\Delta \_n 8$ mutants and $k u 80$. Data are representative of at least three independent experiments. Ctl, naïve mice; *, $\mathrm{P}<0.05$.

(TIF)

Figure S2 Survival of Cyclophosphamide immunosuppressed mice infected with resting conidia of ags $\Delta \_n 8$ mutant and parental $\mathbf{k u} 80$ strains. The survival is expressed in percentage. Data are representative of at least three independent experiments.

(TIF)

Figure S3 Phagocytosis after $1 \mathrm{~h}$ incubation of ags $\Delta \_n 8$ and parental $k u 80$ resting conidia by the macrophages isolated from uninfected mice. Results expressed in number of conidia per macrophages. Data are representative of at least three independent experiments. *, $\mathrm{P}<0.05$.

(TIF)

Figure S4 Imaging and adhesive properties of resting conidia of $\boldsymbol{a g s} \boldsymbol{\Delta} \_\boldsymbol{n \boldsymbol { 8 }}$ mutant. Structural changes correlate with a loss of cell surface adhesive properties. (A) Height images (zrange $=1 \mu \mathrm{m}$; recorded in water with silicon nitride tips); (B) adhesion force maps (z-range: $5 \mathrm{nN}$ ) corresponding to the height image; (C) Representative force-distance curves and adhesion force histograms $(\mathrm{n}=1024)$ recorded on the surface of ags $\Delta \_n 8$ mutant conidia.

(TIF)

Figure S5 NaCl extracted proteins from the surface of the resting ags $\Delta$ triple mutant conidia. SDS-PAGE (10\% gel) of proteins extracted after $2 \mathrm{~h}$ incubation of the resting conidia in $0.5 \mathrm{M} \mathrm{NaCl}$ showing that the three triple ags $\Delta$ mutants (ags $\Delta \_5 T$, ags $\Delta \_n 8$, ags $\left.\Delta \_n 6.2\right)$ displayed the similar protein patterns.

(TIF)

Figure S6 TNFa production or expression by macrophages (isolated from uninfected immunocompetent mice) upon interaction with the parental strain $\mathrm{ku} 80$, ags $\Delta \_n 6.2$ and ags $\Delta \_n 8$ resting conidia, or the ags $\Delta \_n 8$ and ags $\Delta \_n 6.2$ conidial $\mathrm{NaCl}$ extract $(3.2 \mu \mathrm{g}$ proteins) respectively. (A) $\mathrm{TNF} \alpha$ was quantified after $5 \mathrm{~h}$ 
macrophage-conidial interaction. (B) Relative expression of TNF $\alpha$ assessed by real time RT-PGR in total RNA from macrophages after $5 \mathrm{~h}$ incubation of the ags $\Delta \_n 8$ and ags $\Delta \_n 6.2$ conidial $\mathrm{NaCl}$ extract with macrophages. $\mathrm{NaCl}$ supernatant from $k u 80$ resting conidia incubated for $2 \mathrm{~h}$ in $0.5 \mathrm{M} \mathrm{NaCl}$ was used as a control. NS: Non-stimulated. *, $\mathrm{P}<0.05$.

(TIF)

Figure S7 Immunolabeling of Galactosaminogalactan (GAG) and galactomannan (GM) on the swollen conidial surface of the triple ags $\Delta$ mutants and parental $k u 80$ strains. Note that there is no differences in the amount of GAG (A) (labeled by an anti-GAG monoclonal antibody) and GM (B) (labeled by an anti-galf monoclonal antibody) in the triple ags $\Delta$ mutant and parental strains.

(TIF)

Figure S8 Immunolabeling of $\boldsymbol{\alpha}$-(1,3)-glucan. Germinating conidia were labeled with MOPG that recognises $\boldsymbol{\alpha}$-(1,3)-glucan, and mouse TRITC conjugated anti-IgG was used as the

\section{References}

1. Rappleye CA, Eissenberg LG, Goldman WE (2007) Histoplasma capsulatum alpha-(1,3)-glucan blocks innate immune recognition by the beta-glucan receptor. 2007 104: 1366-1370.

2. Reese AJ, Yoneda A, Breger JA, Beauvais A, Liu H, et al. (2007) Loss of cell wall alpha $(1-3)$ glucan affects Cryptococcus neoformans from ultrastructure to virulence. Mol Microbiol 63: 1385-1398.

3. Maubon D, Park S, Tanguy M, Huerre M, Schmitt C, et al. (2006) AGS3, an alpha(1-3)glucan synthase gene family member of Aspergillus fumigatus, modulates mycelium growth in the lung of experimentally infected mice. Fungal Genet Biol 43: 366-375.

4. Beauvais A, Schmidt C, Guadagnini S, Roux P, Perret E, et al. (2007) An extracellular matrix glues together the aerial-grown hyphae of Aspergillus fumigatus. Cell Microbiol 9: 1588-1600.

5. Fontaine T, Beauvais A, Loussert C, Thevenard B, Fulgsang CC, et al. (2010) Cell wall alpha1-3glucans induce the aggregation of germinating conidia of Aspergillus fumigatus. Fungal Genet Biol 47: 707-712.

6. Bozza SC, Cecile; Giovannini, Gloria; Fontaine, Thierry; Beauvais, Anne; Sarfati, Jacqueline; et al (2009) Immune sensing of Aspergillus fumigatus proteins, glycolipids and polysaccharides and the impact on Th immunity and vaccination. The Journal of Immunology 183: 2407-2414.

7. Beauvais A, Maubon D, Park S, Morelle W, Tanguy M, et al. (2005) Two alpha(1-3) glucan synthases with different functions in Aspergillus fumigatus. Appl Environ Microbiol 71: 1531-1538.

8. Henry C, Latge JP, Beauvais A (2012) alphal,3 glucans are dispensable in Aspergillus fumigatus. Eukaryot Cell 11: 26-29.

9. Dague E, Delcorte A, Latge JP, Dufrene YF (2008) Combined use of atomic force microscopy, X-ray photoelectron spectroscopy, and secondary ion mass spectrometry for cell surface analysis. Langmuir 24: 2955-2959.

10. Aimanianda V, Bayry J, Bozza S, Kniemeyer O, Perruccio K, et al. (2009) Surface hydrophobin prevents immune recognition of airborne fungal spores. Nature 460: 1117-1121.

11. Lambou K, Lamarre C, Beau R, Dufour N, Latge JP (2010) Functional analysis of the superoxide dismutase family in Aspergillus fumigatus. Mol Microbiol 75: 910-923.

12. Morelle W, Bernard M, Debeaupuis JP, Buitrago M, Tabouret M, et al. (2005) Galactomannoproteins of Aspergillus fumigatus. Eukaryot Cell 4: 1308-1316.

13. Monod M, Capoccia S, Lechenne B, Zaugg C, Holdom M, et al. (2002) Secreted proteases from pathogenic fungi. Int J Med Microbiol 292: 405-419.

14. Singh B, Oellerich M, Kumar R, Kumar M, Bhadoria DP, et al. (2010) Immuno-reactive molecules identified from the secreted proteome of Aspergillus fumigatus. J Proteome Res 9: 5517-5529.

15. Philippe B, Ibrahim-Granet O, Prevost MC, Gougerot-Pocidalo MA, Sanchez Perez M, et al. (2003) Killing of Aspergillus fumigatus by alveolar macrophages is mediated by reactive oxidant intermediates. Infect Immun 71: 3034-3042.

16. Bayry J, Aimanianda V, Guijarro JI, Sunde M, Latge JP (2012) Hydrophobinsunique fungal proteins. PLoS Pathog 8: e1002700.

17. Sriranganadane D, Waridel P, Salamin K, Reichard U, Grouzmann E, et al. (2010) Aspergillus protein degradation pathways with different secreted protease sets at neutral and acidic pH. J Proteome Res 9: 3511-3519.

18. Wang Z, Lienemann M, Qiau M, Linder MB (2010) Mechanisms of protein adhesion on surface films of hydrophobin. Langmuir 26: 8491-8496.

19. Jimenez-Ortigosa C, Aimanianda V, Muszkieta L, Mouyna I, Alsteens D, et al. (2012) Chitin synthases with a myosin motor-like domain control the resistance of Aspergillus fumigatus to echinocandins. Antimicrob Agents Chemother 56: 6121-6131. secondary antibody. Note the absence of labeling on the triple ags $\Delta$ mutants - ags $\Delta \_5 T$, ags $\Delta \_n 6.2$ and ags $\Delta \_n 8$.

(TIF)

Table S1 Identification of $\mathrm{NaCl}$ extracted conidial surface proteins from the ags $\Delta_{-} 5 T$ and ags $\Delta_{-} 8$ mutants by MALDI-TOF/TOF. ${ }^{(1)}$ Number of peptide peaks identified per protein.

(DOCX)

\section{Acknowledgments}

We thank Maria Pötsch for her excellent technical support.

\section{Author Contributions}

Conceived and designed the experiments: AB OK VB RWR ED MC LR JPL AAB. Performed the experiments: AB SB OK CF VB RWR. Analyzed the data: AB OK VB ED MC LR JPL. Contributed reagents/materials/ analysis tools: AB SB OK VB CH RWR ED. Wrote the paper: AB SB OK VB RWR ED JPL.

20. Alsteens D, Aimanianda V, Hegde P, Pire S, Beau R, et al. (2013) Unraveling the Nanoscale Surface Properties of Chitin Synthase Mutants of Aspergillus fumigatus and Their Biological Implications. Biophys J 105(2):320-7.

21. Hogan LH, Klein BS (1994) Altered expression of surface alpha-1,3-glucan in genetically related strains of Blastomyces dermatitidis that differ in virulence. Infect Immun 62: 3543-3546.

22. Rappleye CA, Goldman GH (2006) Defining virulence genes in the dimorphing fungi. Ann Rev Microbiol 60: 281-303.

23. McDonald JU, Rosas M, Brown GD, Jones SA, Taylor PR (2012) Differential dependencies of monocytes and neutrophils on dectin-1, dectin-2 and complement for the recognition of fungal particles in inflammation. PLoS One 7: e45781.

24. Hohl TM, Feldmesser M, Perlin DS, Pamer EG (2008) Caspofungin modulates inflammatory responses to Aspergillus fumigatus through stage-specific effects on fungal beta-glucan exposure. J Infect Dis 198: 176-185.

25. Lamaris GA, Lewis RE, Chamilos G, May GS, Safdar A, et al. (2008) Caspofungin-mediated beta-glucan unmasking and enhancement of human polymorphonuclear neutrophil activity against Aspergillus and non-Aspergillus hyphae. J Infect Dis 198: 186-192.

26. Latge JP (2010) Tasting the fungal cell wall. Cell Microbiol 12: 863-872.

27. Feriotti C, Loures FV, Frank de Araujo E, da Costa TA, Calich VL (2013) Mannosyl-recognizing receptors induce an M1-like phenotype in macrophages of susceptible mice but an M2-like phenotype in mice resistant to a fungal infection. PLoS One 8: e54845.

28. Fujikawa T, Sakaguchi A, Nishizawa Y, Kouzai Y, Minami E, et al. (2012) Surface alpha-1,3-glucan facilitates fungal stealth infection by interfering with innate immunity in plants. PLoS Pathog 8: e1002882.

29. Roilides E, Dimitriadou-Georgiadou A, Sein T, Kadiltsoglou I, Walsh TJ (1998) Tumor necrosis factor alpha enhances antifungal activities of polymorphonuclear and mononuclear phagocytes against Aspergillus fumigatus. Infect Immun 66: 5999-6003.

30. Roilides E, Uhlig K, Venzon D, Pizzo PA, Walsh TJ (1993) Enhancement of oxidative response and damage caused by human neutrophils to Aspergillus fumigatus hyphae by granulocyte colony-stimulating factor and gamma interferon. Infect Immun 61: 1185-1193.

31. Henriet SS, Hermans PW, Verweij PE, Simonetti E, Holland SM, et al. (2011) Human leukocytes kill Aspergillus nidulans by reactive oxygen speciesindependent mechanisms. Infect Immun 79: 767-773.

32. Zarember KA, Sugui JA, Chang YC, Kwon-Chung KJ, Gallin JI (2007) Human polymorphonuclear leukocytes inhibit Aspergillus fumigatus conidial growth by lactoferrin-mediated iron depletion. J Immunol 178: 63676373 .

33. D'Angelo C, De Luca A, Zelante T, Bonifazi P, Moretti S, et al. (2009) Exogenous pentraxin 3 restores antifungal resistance and restrains inflammation in murine chronic granulomatous disease. J Immunol 183: 4609-4618.

34. Kaur S, Gupta VK, Thiel S, Sarma PU, Madan T (2007) Protective role of mannan-binding lectin in a murine model of invasive pulmonary aspergillosis. Clin Exp Immunol 148: 382-389.

35. Moalli F, Doni A, Deban L, Zelante T, Zagarella S, et al. (2010) Role of complement and $\mathrm{Fc}\{$ gamma\} receptors in the protective activity of the long pentraxin PTX3 against Aspergillus fumigatus. Blood 116: 5170-5180.

36. Speth G, Rambach G (2012) Complement Attack against Aspergillus and Corresponding Evasion Mechanisms. Interdiscip Perspect Infect Dis 2012: 463794 . 
37. Zhang S, Fan Y, Xia YX, Keyhani NO (2010) Sulfonylurea resistance as a new selectable marker for the entomopathogenic fungus Beauveria bassiana. Appl Microbiol Biotechnol 87: 1151-1156.

38. Clavaud C, Beauvais A, Barbin L, Munier-Lehmann H, Latge JP (2012) The composition of the culture medium influences the beta-1,3-glucan metabolism of Aspergillus fumigatus and the antifungal activity of inhibitors of beta-1,3-glucan synthesis. Antimicrob Agents Chemother 56: 3428-3431.

39. Sheth CC, Hall R, Lewis L, Brown AJ, Odds FC, et al. (2011) Glycosylation status of the C. albicans cell wall affects the efficiency of neutrophil phagocytosis and killing but not cytokine signaling. Med Mycol 49: 513-524.

40. Shaler GR, Horvath C, Lai R, Xing Z (2012) Understanding delayed T-cell priming, lung recruitment, and airway luminal T-cell responses in host defense against pulmonary tuberculosis. Clin Dev Immunol 2012: 628293.

41. Svirshchevskaya EV, Shevchenko MA, Huet D, Femenia F, Latge JP, et al. (2009) Susceptibility of mice to invasive aspergillosis correlates with delayed cell influx into the lungs. Int J Immunogenet 36: 289-299.

42. Brian PW, Dawkins AW, Grove JF, Hemming HG, Lowe D, et al. (1961) Phytotoxic compounds produced by Fusarium equiseti. . J Exper Bot 12: 1-12.

43. da Silva Ferreira ME, Kress MR, Savoldi M, Goldman MH, Hartl A, et al. (2006) The akuB(KU80) mutant deficient for nonhomologous end joining is a powerful tool for analyzing pathogenicity in Aspergillus fumigatus. Eukaryot Cell 5: 207-211.

44. Mouyna I, Kniemeyer O, Jank T, Loussert C, Mellado E, et al. (2010) Members of PMT family in Aspergillus fumigatus differentially affect growth, morphogenesis, and viability. Mol Microbiol 76: 1205-1221.

45. Klimpel KR, Goldman WE (1988) Cell walls from avirulent variants of Histoplasma capsulatum lack alpha-(1,3)-glucan. Infect Immun 56: 2997-3000.

46. Harris SD, Morrell JL, Hamer JE (1994) Identification and characterization of Aspergillus nidulans mutants defective in cytokinesis. Genetics 136: 517-532.

47. Dague E, Jauvert E, Laplatine L, Viallet B, Thibault C, et al. (2011) Assembly of live micro-organisms on microstructured PDMS stamps by convective/capillary deposition for AFM bio-experiments. Nanotechnology 22: 395102.

48. Hutter S, Heinritzi K, Reich E, Ehret W (1993) [Effects of different methods of tooth resection in suckling piglets]. Tierarztl Prax 21: 417-428.
49. Bradford M (1976) A rapide and sensitive method for the quantification of microgram quantities of protein utilizing the principle of protein-dye binding. . Anal Biochem 72: 248-254.

50. Kniemeyer O, Lessing F, Scheibner O, Hertweck C, Brakhage AA (2006) Optimisation of a 2-D gel electrophoresis protocol for the human-pathogenic fungus Aspergillus fumigatus. Curr Genet 49: 178-189.

51. Candiano G, Bruschi M, Musante L, Santucci L, Ghiggeri GM, et al. (2004) Blue silver: a very sensitive colloidal Coomassie G-250 staining for proteome analysis. Electrophoresis 25: 1327-1333.

52. Lamarre C, Beau R, Balloy V, Fontaine T, Wong Sak Hoi J, et al. (2009) Galactofuranose attenuates cellular adhesion of Aspergillus fumigatus. Cell Microbiol 11: 1612-1623.

53. Fontaine T, Delangle A, Simenel C, Coddeville B, van Vliet SJ, et al. (2011) Galactosaminogalactan, a new immunosuppressive polysaccharide of Aspergillus fumigatus. PLoS Pathog 7: e 1002372.

54. Stynen D, Sarfati J, Goris A, Prevost MC, Lesourd M, et al. (1992) Rat monoclonal antibodies against Aspergillus galactomannan. Infect Immun 60: 2237-2245.

55. Mishima Y, Quintin J, Aimanianda V, Kellenberger C, Coste F, et al. (2009) The N-terminal domain of Drosophila Gram-negative binding protein 3 (GNBP3) defines a novel family of fungal pattern recognition receptors. J Biol Chem 284: 28687-28697.

56. Takagi I, Yamada K, Sato T, Hanaichi T, Iwamoto T, et al. (1990) J Electron Microsc (Tokyo) 39: 67-68.

57. Bonifazi P, D'Angelo C, Zagarella S, Zelante T, Bozza S, et al. (2010) Intranasally delivered siRNA targeting P13K/Akt/mTOR inflammatory pathways protects from aspergillosis. Mucosal Immnunol 3: 193-205.

58. Bozza S, Perruccio K, Montagnoli C, Gaziano R, Bellocchio S, et al. (2003) A dendritic cell vaccine against invasive aspergillosis in allogeneic hematopoietic transplantation. Blood 102: 3807-3814.

59. Sturtevant J, Latge JP (1992) Participation of complement in the phagocytosis of the conidia of Aspergillus fumigatus by human polymorphonuclear cells. J Infect Dis 166: $580-586$. 